\title{
Spectral Statistics on Zoll Surfaces
}

\author{
Alejandro Uribe ${ }^{1 \star}$ and Steven Zelditch ${ }^{2 \star \star}$ \\ 1 Mathematics Department, University of Michigan, Ann Arbor, MI 48109, and Institute for \\ Advanced Study, Princeton, NJ 08540, USA \\ 2 Mathematics Department, Johns Hopkins University, Baltimore, MD 21218, USA
}

Received June 15, 1992; in revised form November 23, 1992

\section{Contents}

1. Level Spacings and Correlation Functions $\quad 314$

2. Spectrum in the Completely Integrable Case 318

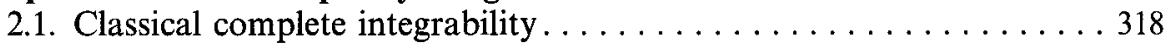

2.2. Quantum integrability. . . . . . . . . . . . . . . . . 321

2.3. Some conjectures and results on the lsd and kcf's . . . . . . . . 322

3. Pair Correlation of a Zoll Metric 324

3.1. Statement of the result . . . . . . . . . . . . . . . 324

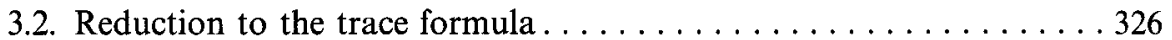

3.3. Period manifolds and holonomies. . . . . . . . . . . . . 328

3.4. The pair correlation density . . . . . . . . . . . . . . . . . . . . 329

3.4 .1 The trace formula for periodic flows $\ldots \ldots \ldots 330$

3.4 .2 Computation of the pcf $\ldots \ldots \ldots \ldots \ldots \ldots \ldots \ldots \ldots \ldots$

4. The Trace Formula for Commuting Operators 333

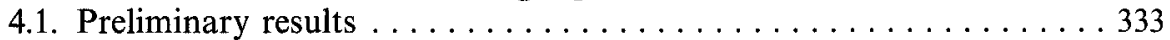

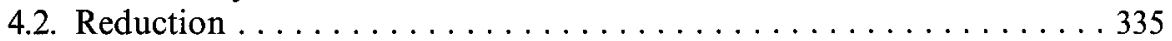

4.3. Conclusions. . . . . . . . . . . . . . . . 336

5. $\boldsymbol{k}$-level Correlation Functions 338

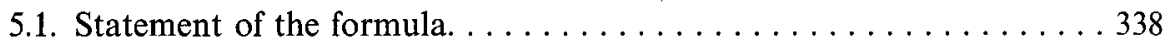

5.2. Reduction to the trace formula . . . . . . . . . . . . . . . 339

5.3. The end of the proof . . . . . . . . . . . . . . . . 340

6. On the Level Spacings Distribution 344

\footnotetext{
* Research supported by NSF grants DMS-8907997 and DMS 8610730

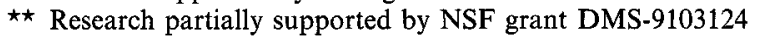


Abstract. The subject of this article is a family of (partly conjectural) spectral invariants of a Laplace operator with a discrete spectrum $\lambda_{j}$ known as its $k$-level correlation functions $\rho_{k}$ and level spacings distribution $v$. The 2-level (or pair) correlation function $\rho_{2}$, for instance, measures the asymptotic number of spacings $\lambda_{i}^{*}-\lambda_{j}^{*}$ between normalized eigenvalues $\lambda_{i}^{*}$ as the eigenvalues tend to infinity. The normalization is so that the mean level spacing $\lambda_{i+1}^{*}-\lambda_{i}^{*}$ is one. These invariants have been studied by many physicists, including Wigner, Dyson, and Berry, and are conjectured to have certain universal forms depending on the qualitative behaviour of the corresponding classical (geodesic) flow. In particular, when the flow is a generic completely integrable system, these invariants are conjectured to conform to Poisson statistics.

Our main result is an explicit formula for these invariants for the Laplacian on a Zoll sphere: that is, for a metric on the 2-sphere all of whose geodesics are closed. More generally, we can determine these invariants for a Schrodinger operator on the sphere. Our results show that they are determined from a certain Hamiltonian flow on the space of geodesics of the Zoll sphere (exactly for these metrics, the space of geodesics is a well defined manifold).

Although Zoll metrics have very special properties, they form the largest class of surface metrics with completely integrable geodesic flow. Their Laplacians also appear to be the first (infinite dimensional) family of operators for which one can determine the above spectral invariants. Our methods suggest that in general the nature (even the existence) of these invariants is closely related to the noncommutative geometry of the geodesic foliation of $S^{*} M$. In the Zoll case, the geometry is commutative (in the sense of A. Connes). In all other cases, the geometry is noncommutative and our methods seem to lead into correspondingly nonclassical kinds of Fourier Integral Operators.

\section{Level Spacings and Correlation Functions}

We begin by defining these invariants in the general context of compact Riemannian manifolds.

First, recall that from the spectral point of view there is a gross dichotomy among metrics: (1) the Zoll case considered here (where $(M, g)$ has periodic geodesic flow), and (2) the generic case, where the set of closed geodesics has measure zero. In the Zoll case, the eigenvalues of the square root $\sqrt{\Delta}$ of $\Delta$ cluster around an arithmetic progression

$$
\left\{\frac{2 \pi}{T} k+\beta ; k=0,1,2, \ldots\right\}
$$

where $T$ is the period and $\beta$ is closely related to the common Morse index of the periodic geodesics of period $T([16,10])$. More precisely, if $0=\lambda_{0}<\lambda_{1} \leqq \lambda_{2} \leqq \cdots$ denote the eigenvalues of $\Delta$, then the eigenvalues $\left\{\sqrt{\lambda_{j}}\right\}$ lie in intervals of size $O(1 / k)$ around the $\left\{\frac{2 \pi}{T} k+\beta\right\}$. In the generic case, the eigenvalues of $\sqrt{\Delta}$ are more uniformly distributed: in particular, the number $N(\lambda, \varepsilon)$ of eigenvalues $\sqrt{\lambda_{j}}$ in $[\lambda-\varepsilon, \lambda+\varepsilon]$ is asymptotically $C_{n} 2 \varepsilon \lambda^{n-1} \operatorname{Vol}(M)(n$ being the dimension of $M$ ). 
Our definitions of the $k$-level correlation functions (kcf's) and level spacings distribution (lsd) correspondingly differ a little between these two cases. The general idea $([14,25,30])$ is to measure the limit distribution of normalized spacings between eigenvalues of $\sqrt{\Delta}$ in $[\lambda-\varepsilon, \lambda+\varepsilon]$ as $\lambda \rightarrow \infty$. By "normalized" we mean that the eigenvalues are rescaled to have a fixed mean level spacing independent of $\lambda$. In the generic case, the mean level spacing of the eigenvalues in $[\lambda-\varepsilon, \lambda+\varepsilon]$ is evidently

$$
D_{\lambda}=\frac{1}{N(\lambda, \varepsilon)} \sum\left(\sqrt{\lambda_{j+1}}-\sqrt{\lambda_{j}}\right) .
$$

(The sum runs over $\sqrt{\lambda_{j}} \in[\lambda-\varepsilon, \lambda+\varepsilon]$.) In the generic case,

$$
D_{\lambda} \sim C \cdot \frac{1}{\lambda^{n-1}},
$$

where $C=\left(C_{n} \operatorname{Vol}(M)\right)^{-1}$. Henceforth we will rescale the metric so that $C=1$. Thus, the rescaled eigenvalues $D_{\lambda}^{-1}\left(\sqrt{\lambda_{j}}\right)$ have unit mean level spacing. We now define the pair correlation functions (pcf's), $\rho_{2}^{\lambda}$ of the normalized eigenvalues in this interval by:

\section{Definition 1.1.}

$$
\rho_{2}^{\lambda}=\frac{1}{N(\lambda, \varepsilon)} \sum_{i, j} \delta_{D_{\lambda}^{-1}\left(\sqrt{\lambda_{i}}-\sqrt{\lambda_{j}}\right)}
$$

where as usual the sum runs over $\sqrt{\lambda_{i}}, \sqrt{\lambda_{j}} \in[\lambda-\varepsilon, \lambda+\varepsilon]$. Thus, $\rho_{2}^{\lambda}$ is a measure on $\mathbb{R}$ with $\delta$-functions at each normalized spacing. Note that $\rho_{2}^{\lambda}$ is not of mass one, or even of bounded mass (in $\lambda$ ). Hence, there is no guarantee that the family $\left\{\rho_{2}^{\lambda}\right\}$ has any limit points, much less a unique one, nor that a limit is a measure on $\mathbb{R}$. Nevertheless, one can ask what the limits are. We will denote any such limit by $\rho_{2}$, and call it a pcf.

The lsd is defined in a similar way except that we only sum over nearest neighbors: $i=j+1$. The resulting measures, say $\left\{v_{\lambda}\right\}$, are then of mass one, so there surely are weak limit measures. We will denote any one by $v$, and refer to it as an lsd.

The kcf's for $k>2$ are in general measures on $\mathbb{R}^{k-1}$. For each $k$-tuple $\left(\sqrt{\lambda_{i_{1}}}, \ldots, \sqrt{\lambda_{i_{k}}}\right)$ of eigenvalues in $[\lambda-\varepsilon, \lambda+\varepsilon]$, we put a $\delta$-function at the point

$$
D_{\lambda}^{-1}\left(\sqrt{\lambda_{i_{2}}}-\sqrt{\lambda_{i_{1}}}, \ldots, \sqrt{\lambda_{i_{k}}}-\sqrt{\lambda_{i_{k-1}}}\right)
$$

in $\mathbb{R}^{k-1}$. The $\mathrm{kcf}, \rho_{k}^{\lambda}$, for $[\lambda-\varepsilon, \lambda+\varepsilon]$ is then:

\section{Definition 1.2.}

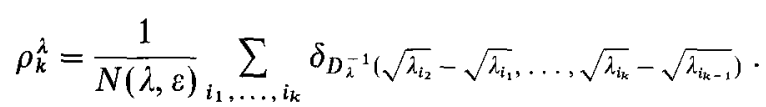

Any weak limit, $\rho_{k}$, of the family $\left\{\rho_{k}^{\lambda}\right\}$ will be called a limit kcf.

In the Zoll case studied here, these definitions $(1.1,1.2)$ need to be modified to insure the existence of a unique weak limit. To see this, we recall that by Weinstein's 
theorem, [29], the Zoll Laplacian is unitarily equivalent to $\Delta_{0}+A$ for some zeroth order $\Psi D O, A$ :

$$
F^{-1} \Delta F=\Delta_{0}+A .
$$

Moreover, $A$ can be assumed to commute with the standard Laplacian, $\Delta_{0}$, on $S^{2}$ [17]. Let

$$
A_{l}=\left.A\right|_{H_{l}},
$$

where $H_{l}$ is the space of spherical harmonics of degree $l$. Then $A_{l}$ is a $(2 l+1) \times(2 l+1)$ Hermitian matrix, with $2 l+1$ eigenvalues which will be denoted $\left\{\mu_{l, k}\right\}$. Clearly

$$
\left|\mu_{l, k}\right| \leqq\|A\|,
$$

and so the spectrum of $\Delta$ comes in the bounded clusters

$$
C_{l}=\left\{l(l+1)+\mu_{l, k} ; k=1, \ldots, 2 l+1, l=1,2, \ldots\right\} .
$$

Therefore the spectrum of $\sqrt{\Delta}$ comes in the clusters

$$
C_{l}^{1 / 2}=\left\{l+1 / 2+l^{-1}\left(\mu_{l, k}-1 / 4\right)+O\left(l^{-2}\right) ; k=1, \ldots, 2 l+1, l=1,2, \ldots\right\} .
$$

Since these clusters are of size $O\left(l^{-1}\right)$, and are separated by intervals of size $1-O(1 / l)$, there will be no unique lsd or kcf's in the sense of Definitions 1.1, 1.2. However, there is clearly only one interesting limit, obtained by fixing

$$
\lambda=l+\frac{1}{2} .
$$

In other words, we will define the lsd of $\sqrt{\Delta}$ (e.g.) as the limit 1sd of the clusters $C_{l}^{1 / 2}$.

Since the width of $C_{l}^{1 / 2}$ is $O(1 / l)$, and since there are $2 l+1$ eigenvalues in it, the mean level spacing $D_{l}$ of the cluster is $O\left(1 / l^{2}\right)$. The implicit constant in $D_{l}$ can be fixed as follows: According to [28], the eigenvalues $\left\{\mu_{l, k}\right\}$ have a limit distribution as $l \rightarrow \infty$. To describe it, let $\sigma_{A}$ be the principal symbol of $A$. Since $\left[A, \Delta_{0}\right]=0, \sigma_{A}$ is invariant under the geodesic flow of the standard metric $g_{0}$ on $S^{2}$. Hence, it descends to the symplectic quotient

$$
\mathcal{O}=S^{*}\left(S^{2}\right) / S^{1},
$$

where $S^{1}$ acts by the geodesic flow of $g_{0}$. Let $\omega_{0}$ be the symplectic volume form of 0 . Then Weinstein's result is that the push-forward $\left(\sigma_{A}\right) * \omega_{\mathcal{O}}$ is the piecewise smooth measure $\mu_{A}$ on the interval $\left[\min \sigma_{A}, \max \sigma_{A}\right]$ according to which the $\left\{\mu_{l, k}\right\}$ get distributed. Since all but a sparse subsequence of the $\mu_{l, k}$ 's must lie in $\left[\min \sigma_{A}, \max \sigma_{A}\right]$, it seems sensible to define:

$$
D_{l}=\frac{\max \sigma_{A}-\min \sigma_{A}}{2 l+1} .
$$

By a little rescaling, we will henceforth assume $D_{l}=1 / l$.

We therefore set: 
Definition 1.3. In the Zoll case,

$$
\begin{aligned}
\rho_{2}^{l} & =\frac{1}{2 l+1} \sum_{i j} \delta_{l\left(\mu_{l, i}-\mu_{l, j}\right)}, \\
v_{2}^{l} & =\frac{1}{2 l+1} \sum_{i} \delta_{l\left(\mu_{l, i+1}-\mu_{l, i}\right)}, \\
\rho_{k}^{l} & =\frac{1}{2 l+1} \sum_{i_{1}, \ldots, i_{k}} \delta_{l\left(\mu_{l, i_{2}}-\mu_{l, i_{1}}, \ldots, \mu_{l, i_{k}}-\mu_{l_{i, i_{k}-1}}\right) .}
\end{aligned}
$$

Hence, we take advantage of the fact that terms of order $O\left(l^{-2}\right)$ in the formulae for the eigenvalues of $\sqrt{\Delta}$ will drop out in the limit.

We finish this section with some general remarks that motivate our approach to the pair correlation function of an elliptic operator. (Suitable generalizations apply to the kcf.) Let $P$ be a positive, self-adjoint, first-order elliptic pseudodifferential operator on a compact manifold $M$, and let $\mu_{1} \leqq \mu_{2} \leqq \ldots$ denote its eigenvalues with multiplicities. Define the operator

$$
Q=P \otimes I-I \otimes P
$$

on functions on $M \times M$. Then the differences of pairs of eigenvalues

$$
\mu_{j}-\mu_{k}, \quad j, k \geqq 1
$$

are clearly eigenvalues of $Q$, and thus by a theorem of Helton the operator $Q$ will generically have dense point spectrum. $Q$ is a pseudodifferential operator of order one on $M \times M$, with the minor complication that its symbol $\sigma_{Q}$ is non-smooth on $(\{0\} \times X) \cup(X \times\{0\})$, where $X=T^{*} M \backslash\{0\}$. In the informal discussion that follows we will safely ignore this fact and treat $Q$ as an ordinary first-order, selfadjoint pseudodifferential operator of real principal type. The latter means that (a) zero is a regular value of the principal symbol of $P$, and (b) at any point of the manifold $Y$,

$$
Y=\sigma_{Q}^{-1}(0)=\left\{(x, \xi ; y, \eta) ;|\xi|_{x}=|\eta|_{y}\right\},
$$

the Hamilton vector field of $\sigma_{Q}$ is non-radial. Notice that, by the ellipticity of $P, Y$ does not intersect the set $(\{0\} \times X) \cup(X \times\{0\})$, where $\sigma_{Q}$ is non-smooth.

The flow of the Hamilton vector field of $\sigma_{Q}$ on $Y$ (the null bicharacteristic flow of $Q$ ) is obviously given by

$$
(x, \xi ; y, \eta) \mapsto\left(\phi_{t}(x, \xi) ; \phi_{-t}(y, \eta)\right),
$$

where $\phi_{t}$ denotes the Hamilton flow of the symbol of $P$ on $X$.

Thus by the introduction of the operator $Q$ we can cast the problem of the pair correlation function as a particular case of the following problem: How does the null bicharacteristic flow of an operator of real principal type describe the asymptotic distribution of its small eigenvalues? (In cases of interest one has a commuting operator, $P$, jointly elliptic with $Q$, which orders the small eigenvalues of $Q$ and makes this problem precise.) 
Theorem 4.1 of [8] says that if $Q$ is any first-order self-adjoint operator of real principal type whose null bicharacteristic flow is periodic, then a suitable perturbation of $Q$ has an infinite-dimensional kernel, $\mathscr{H}_{Q}$. (More precisely, it has such a kernel in the FIO category: i.e. some projection into the kernel is an FIO.) In some sense, $\mathscr{H}_{Q}$ is the quantization of the symplectic manifold quotient of the bicharacteristic variety of $Q$ by its null flow. In case $Q$ is of the form (3), this theorem implies the clustering results of Duistermaat-Guillemin/Colin de Verdiere/Weinstein: if the flow $\phi_{t}$ is periodic, the eigenvalues $\mu_{j}$ cluster around an arithmetic sequence.

In the non-periodic case, the situation is a great deal more complicated. First, the orbit space of the null bicharacteristic flow on $\operatorname{ch}(Q)$ (the characteristic variety of $Q$ ) need not exist as a Hausdorff topological space, much less as a manifold. Secondly, there need not exist a quantizing Hilbert space $\mathscr{H}_{Q}$ either. Indeed, an (FIO) projection $\Pi$ to $\mathscr{H}_{Q}$ would have to lie in the algebra $\mathscr{R}_{\mathrm{ch}(Q)}$ associated to $\operatorname{ch}(Q)$ by Guillemin and Sternberg in [19]. But $\mathscr{R}_{\mathrm{ch}(Q)}$ might not have any projections. To see this, we observe (following Guillemin-Uribe in [23]) that the symbol calculus of $\mathscr{R}_{\mathrm{ch}(Q)}$ is essentially the Connes $C^{*}$ algebra of the null foliation of $\mathscr{R}_{\mathrm{ch}(Q)}$. For some foliations (for example the horocycle foliation of a compact quotient of $S L(2, \mathbb{R})$ ), this $C^{*}$ algebra is known to have no projections, [13]. In general, the existence of projections in this algebra is a difficult question.

Even when a projection exists, the problem of determining the asymptotics of $\rho_{2}^{\lambda}$ seems to be quite non-standard. The novelty stems from the eigenvalue rescaling that goes into the definition of $\rho_{2}^{\lambda}$. It amounts to replacing $Q$ by $R Q$, where $R$ is a $\Psi \mathrm{DO}$ of $\operatorname{order}(n-1)$. The asymptotics of $\rho_{2}^{\lambda}$ then reduces to the asymptotics of the traces $\operatorname{Tr} \Pi \phi_{\lambda}(R Q)$, for suitable test functions $\phi_{\lambda}$. But $\Pi \phi_{\lambda}(R Q)$ is not an FIO in the classical sense, since the order of $R Q$ is greater than one. The $\Pi$ factor has the effect of reducing the order of $R Q$ by one (since the symbol of $Q$ vanishes on the canonical relation of $\Pi$ ). Hence if $n=2, \Pi \phi_{\lambda}(R Q)$ has order one, in the sense of the non-commutative symbol algebra of $\mathscr{R}_{\operatorname{ch}(Q)}$.

In sum, the problem of determining the asymptotics of $\rho_{2}^{\lambda}$ in general leads to a number of new, and subtle, problems in non-commutative microlocal analysis.

\section{Spectrum in the Completely Integrable Case}

We now briefly review what is known (rigorously) about the spectrum of $(M, g)$ under various conditions of complete integrability. We will also review some well-known conjectures and partial results, especially as regards the lsd and kcf's. In the rest of the paper we will discuss in more detail the special case of Zoll surfaces.

2.1. Classical Complete Integrability. Let $q(x, \xi)=|\xi|$ denote the norm function of the metric $g$, viewed as a function on $T^{*} M$. The flow generated by the Hamilton vector field $\Xi_{q}$ is the geodesic flow and will be denoted $G^{t}$. It is completely integrable if there is a smooth, positively homogeneous map

$$
f: T^{*} M \backslash\{0\} \rightarrow \mathbb{R}^{n} \backslash\{0\}
$$


$(n=\operatorname{dim} M)$ satisfying:

$$
\begin{aligned}
f_{1} & =q, \\
\forall i, j \quad\left\{f_{i}, f_{j}\right\} & =0, \\
d f_{1} \wedge \cdots \wedge d f_{n} & \neq 0,
\end{aligned}
$$

where the third condition should hold on some open dense set $\Omega_{r}^{h}$, the set of regular points of $f$.

The collection $\left\{f_{j}\right\}$ of commuting Hamiltonians generates an $\mathbb{R}^{n}$ action, $\Phi^{t}, t=\left(t_{1}, \ldots, t_{n}\right)$, on $T^{*} M \backslash\{0\}$. Since all the $f_{j}$ 's are homogeneous of degree one, $\Phi^{t}$ is homogeneous of degree zero, and so it restricts to an action on the unit sphere bundle, $S^{*} M=q^{-1}(1)$. We let $\Omega_{r}=\Omega_{r}^{h} \cap S^{*} M$.

The decomposition of $S^{*} M$ into orbits of $\Phi^{t}$ defines a foliation with singularities along $\Omega_{s}=S^{*} M \backslash \Omega_{r}$. The foliation is regular along $\Omega_{r}$, and the leaves are of the form $\mathbb{R}^{n} / \Gamma_{(x, \xi)}$, where $\Gamma_{(x, \xi)}$ is the isotropy subgroup at $(x, \xi)$. Since, by definition, the Hamilton vector fields of the $f_{j}$ are linearly independent at each point of $\Omega_{r}$, the isotropy subgroups $\Gamma_{(x, \xi)}$ are discrete. Let us denote by $\Omega_{c}$ the subset of $\Omega_{r}$ such that $\Gamma_{(x, \xi)}$ is co-compact, i.e. so that the orbit through $(x, \xi)$ is an $n$-torus. $\Omega_{c}$ is an open subset of $\Omega_{r}$, and $f: \Omega_{c} \rightarrow A$ (where $A=f\left(\Omega_{c}\right)$ is a proper submersion onto an open set in $\mathbb{R}^{n}$, whose inverse images $f^{-1}(b)$ are finite unions of $n$-torii. In particular, if $\Omega_{c}$ is connected. $\left.f\right|_{\Omega_{c}}$ is a fibration. The same holds for the homogeneous set $\Omega_{c}^{h}=\mathbb{R}^{+} \Omega_{c}$.

The well-known Bohr-Sommerfeld-Maslov (or BSM) quantization rule selects from among the invariant torii $\Lambda_{k}$ of $\Omega_{c}^{h}$ those satisfying

$$
\frac{1}{2 \pi} \int_{\gamma_{j}} \alpha-1 / 4 m\left(\gamma_{j}\right)=k_{j} \in \mathbb{Z},
$$

where $\left\{\gamma_{1}, \ldots, \gamma_{n}\right\}$ is a basis for $H_{1}\left(\Lambda_{k}, \mathbb{Z}\right)$ and $m\left(\gamma_{j}\right)$ denotes the Maslov index, [11]. The discrete set $\left\{\Lambda_{k}\right\}$ of such quantizable torii is important because, at least heuristically, it corresponds to an orthonormal basis of eigenfunctions of the Laplacian. Further, the quasi-classical spectrum

$$
\operatorname{QSpec}(\sqrt{\Delta})=\left\{q\left(\Lambda_{k}\right) ; k \in \mathbb{Z}^{n}\right\}=\left\{0=\mu_{0}<\mu_{1} \leqq \mu_{2} \ldots \rightarrow \infty\right\}
$$

is supposed to provide a first approximation to the true spectrum of $\sqrt{\Delta}$. By adding lower order terms to $q$, it is supposed to provide an arbitrarily good approximation of the spectrum of $\sqrt{\Delta}$. In particular, the lsd and $\mathrm{kcf}$ of $\operatorname{Spec} \sqrt{\Delta}$ are supposed to coincide with those of $\operatorname{QSpec}(\sqrt{\Delta})$.

To substantiate this picture, one must construct a bijection

$$
\mu_{k} \leftrightarrow \lambda_{j(k)}
$$

between quasi-classical and true eigenvalues so that $\left|\mu_{k}-\lambda_{j(k)}\right|$ is of lower order than the mean level spacing near $\sqrt{\lambda_{j(k)}}$. (Actually, initial eigenvalues $\left\{\mu_{j} ; j \leqq N\right\}$ resp. $\left\{\lambda_{j} ; j \leqq N\right\}$ can be ignored without compromising any principles.) In general, however, there is no natural definition of $j$. Specifically, in this approach one must solve the following problems:

(a) The Approximation Problem. Is there an injection $j$ from the quasi-classical spectrum $\left\{\tau_{r}\right\}$ to spec $\sqrt{\Delta}$ so that $\left|j\left(\tau_{r}\right)-\tau_{r}\right|=o\left(D_{\tau_{r}}\right)$, where $D_{\tau_{r}}$ is the mean level spacing near $\tau_{r}$ ? 
(b) The Exhaustion Problem. Assuming one has constructed $j$ as in (a), does the image of $j$ contain a cofinal set $\left\{\lambda_{j} ; j \geqq N\right\}$ ? (A weaker condition is that the image of $j$ is a set of spectral density one.)

The standard approach to these problems is by the construction of quasimodes. We now recall the notion (discussed in [11]) of a quasi-mode.

Definition 2.1 [11]. A quasi-mode of order $\sigma \in \mathbb{R}^{+} \cup\{\infty\}$ is a sequence of pairs $\left(u_{r}, \tau_{r}\right)$ where:

$$
\tau_{0} \leqq \tau_{1} \leqq \tau_{2} \leqq \cdots \rightarrow \infty
$$

tends monotonically to infinity, and

$$
\left\|\left(\Delta-\tau_{r}\right) u_{r}\right\|=O\left(\tau_{r}^{-\sigma / 2}\right) .
$$

Thus a quasi-mode is a sequence of approximate eigenfunctions and approximate eigenvalues. The main significance of a quasi-mode is that $\left\{\tau_{\boldsymbol{r}}\right\}$ are indeed approximate eigenvalues: there is an increasing function $j: r \mapsto j_{r}$ of $\mathbb{N}$ into itself such that

$$
\lambda_{j_{n}}=\tau_{r}+O\left(\tau_{r}^{-\sigma / 2}\right)
$$

([11], Cor. (1.4)). However, the $u_{r}$ do not necessarily approximate true eigenfunctions in $L^{2}(M)$, [1]. Rather, they are associated to quantizable torii:

Theorem 2.2 ([11], Theorem 7.1; [15] §1.5). Let $A \subset T^{*} M \backslash\{0\}$ be a Lagrangian torus, contained in $S^{*} M$ and satisfying:

(i) A generalized Maslov quantization condition: If $[\xi d x] \in H^{1}(\Lambda, \mathbb{R})$ is the class of the pull-back to $\Lambda$ of the canonical 1 -form, and $\alpha \in H^{1}(\Lambda, \mathbb{Z})$ is the Maslov class, then there exists a sequence $\left\{n_{r}\right\} \subset \mathbb{R}^{+}$so that $\left|n_{r+1}-n_{r}\right| \geqq C>0$ and so that

$$
\text { distance }\left(\frac{n_{r}}{2 \pi}[\xi d x]-\alpha / 4, H^{1}(\Lambda, \mathbb{Z})\right)=O\left(1 / n_{r}\right) \text {. }
$$

(ii) For any $f \in C^{\infty}(\Lambda)$ such that $\int_{\Lambda} f d \mu_{\Lambda}=0$ (where $d \mu_{\Lambda}$ is Liouville measure), there exists $g \in C^{\infty}(\Lambda)$ such that $H_{q}(g)=f$.

Then: There is a quasi-mode $\left\{\left(u_{r}, \tau_{r}\right)\right\}$ of order $\infty$ so that

$$
\tau_{r} \sim n_{r}^{2}+\sum_{j=0}^{\infty} P_{j}\left(\beta_{r, 1}, \ldots, \beta_{r, n}\right) n_{r}^{-j},
$$

where we have written

$$
\frac{n_{r}}{2 \pi}[\xi d x]-\alpha / 4=\frac{1}{2 \pi n_{r}} \sum_{i=1}^{n} \beta_{r, i} e_{i}
$$

with $\left\{e_{i}\right\}$ a basis of $H^{1}(\Lambda, \mathbb{R}), \beta_{r, i}=O(1)$ and $P_{j}$ is a polynomial of degree $(j+1)$ in $n$ variables.

Without condition (ii), one can only construct a quasi-mode of order zero, i.e. $\lambda_{j_{r}}=n_{r}^{2}+O(1)$. Such a quasi-mode is of no use in the study of lsd's or kcf's, since $\left|\lambda_{j_{r}}-n_{r}^{2}\right|$ is not of smaller order than the mean level spacing. Also, as Colin points out, condition (ii) only holds if each geodesic in $A$ is dense. 
The correspondence between quantizable torii and quasi-modes can be strengthened to encompass the asymptotics of the $\left\{u_{r}\right\}$. The idea is that the $u_{r}$ micro-locally concentrate on $A$ as $r \rightarrow \infty$ (or: $A$ is the micro-support of the quasi-mode), [11]. In contrast, there is generally no such natural correspondence between modes (actual eigenfunctions) and quasi-modes [1].

Theorem (2.2) cited above gives one solution to the approximation problem (a). A second approach is to use intertwining operators to microlocal normal forms, ([12]). However, this only seems to construct quasi-modes of order zero, so it does not solve the approximation problem accurately enough.

As for the exhaustion problem (b), the only approach in general is to count the number

$$
N_{S C}(T)=\#\left\{r ; \tau_{r} \leqq T\right\}
$$

of quasi-classical eigenvalues less than $T$, and show it has the same growth rate as $N(\lambda)$. This at least confirms the weaker form of (b). $N_{S C}$ is evaluated by decomposing $S^{*} M$ into open subsets $\left\{\Omega_{i}\right\}$ on which action-angle variables $\chi_{i}$ can be defined. BMS torii $\Lambda_{k}$ in $\Omega_{i}^{h}$ are distinguished in that the action variables $\left[I_{i, 1}, \ldots, I_{i, n}\right]$ take $\Lambda_{k}$ to $k+\alpha / 4$, so essentially convert the BMS torii to the lattice $\mathbb{Z}^{n} \cap \chi_{i}\left(\Omega_{i}^{h}\right)$. Thus, counting BMS torii $\Lambda_{k}$ in $\Omega_{i}^{h}$, with $q\left(\Lambda_{k}\right) \leqq T$, is converted to counting lattice points in $\chi_{i}\left(\Omega_{i}^{h}\right)$ with $K_{i}:=q \circ \chi_{i} \leqq T$. This is a classical van der Corput problem if $K_{i}^{\prime \prime}$ is of maximal rank; the number of lattice points then grows like the volume of

$$
\left\{(x, \xi) \in \Omega_{i}^{h} ; q(x, \xi) \leqq T\right\} .
$$

Thus, one gets the optimal growth rate for the BSM spectrum in $\Omega_{i}^{h}$. If the $\Omega_{i}^{h}$ were disjoint and the complement of their union had measure zero, one would like to conclude that

$$
\frac{N_{S C}(T)}{N(T)} \rightarrow L .
$$

This, however, is a difficult programme to implement. First, while the existence of such a decomposition may be possible in general, no proof of it seems to exist in the literature. To our knowledge, the best available result only uses the existence of a decomposition of $S^{*} M$ up to measure $\varepsilon([11]$, p. 32). Secondly, the van der Corput problem alluded to above becomes singular where $K_{i}^{\prime \prime}$ degenerates, this giving rise to a higher concentration of eigenvalues (see [9]). Hence, the exhaustion problem is unresolved in general.

2.2. Quantum Integrability. In spectral theory, and in this paper, one is usually dealing with the more specialized situation of a quantum completely integrable system. This means $\sqrt{\Delta}=P_{1}$ is one in a collection of commuting first-order $\Psi$ DO's, $P_{1}, \ldots, P_{n}$ : $\left[P_{i}, P_{j}\right]=0 \forall i, j$. (This setting also covers the apparently more general small $\hbar$ setting for operators $\hbar$-admissibles, as in [26].) The principal symbols $\left(p_{1}, \ldots p_{n}\right)$ are assumed to be a.e. independent, and hence define a classical completely integrable system. But the additional assumption that the $p_{i}$ are symbols of commuting YDO's leads to somewhat stronger conclusions about the spectrum of $\Delta$ than before.

The strongest conclusions can be drawn if the $\left\{P_{i}\right\}$ come from the infinitesimal representation of a quantum torus action: i.e. a representation of $t \in \mathbb{R}^{n} / \mathbb{Z}^{n}$ as 
unitary FIO's (Fourier integral operators), $U(t)$, on $L^{2}(M)$. This always holds if classically the $\left\{p_{i}\right\}$ come from a closed torus action. See [12] $\S 2$, and [18] for discussions of conditions under which a completely integrable system is a Hamilton torus action, and [12] for a proof that such an action can be quantized. Following Colin's paper, we let $q=\left(q_{1}, \ldots q_{n}\right)$ denote the infinitesimal generators of the torus action so that the flow of $H_{q_{i}}$ is periodic of period $2 \pi$, and let $\left(Q_{1}, \ldots Q_{n}\right)$ denote generators of the corresponding commutative algebra of $\Psi$ DO's so that the joint spectrum

$$
\operatorname{Spec}\left(Q_{1}, \ldots, Q_{n}\right) \subset \mathbb{Z}^{n}+\mu,
$$

where $\mu$ is the Maslov index.

Theorem 2.3 ([12], Theorem 3.2).

(i) There exists $R>0$ such that

$$
\operatorname{Spec}\left(Q_{1}, \ldots, Q_{n}\right) \cap\{\|\lambda\|>R\}=q\left(T^{*} M \backslash\{0\}\right) \cap\{\|\lambda\|>R\} \cap \mathbb{Z}^{n}+\mu,
$$

(ii) Each joint spectral point $\lambda$ with $\|\lambda\|>R$ has multiplicity one.

Thus, up to a finite number of possible exceptions, the BMS torii $q^{-1}(\lambda)$ are in one to one correspondence with $\operatorname{Spec}\left(Q_{1}, \ldots, Q_{n}\right)$, and hence both the approximation and the exhaustion problems can be optimally solved.

Unfortunately, few quantum integrable systems are globally quantum torus actions. In particular, the Zoll surface system is generally not. However, the existence of $\left\{P_{1}, \ldots, P_{n}\right\}$ at least allows the eigenvalues of $P_{1}=\sqrt{\Delta}$ to be separated out: i.e. the joint spectrum $\left(\sqrt{\delta}, \mu_{2}, \ldots, \mu_{n}\right)$ of $\left\{P_{1}, \ldots, P_{n}\right\}$ can be widely separated even though the first coordinates $\sqrt{\delta}$ are not. This of course depends on the choice of generators for the infinitesimal torus action.

In good cases the joint spectral points can be put in a single 1-1 correspondence with the quasi-classical joint spectrum of values $\left(p_{1}\left(\Lambda_{k}\right), \ldots, p_{n}\left(\Lambda_{k}\right)\right)$, but no definitive statement seems to exist in the literature. When $S^{*} M$ can be decomposed into a finite number $\Omega_{i}$ of invariant open sets, such that $S^{*} M \backslash \bigcup_{i} \Omega_{i}$ has measure zero and one has toral actions on each $\Omega_{i}$, one is tempted to try and apply Theorem (2.3) to each $\Omega_{i}$ and conclude that the exhaustion problem is solved. However, the joint spectrum will be in general highly concentrated along the boundaries of the $q\left(\Omega_{i}\right)$, and such a conclusion is at least not at all clear.

2.3. Some Conjectures and Results on the lsd and kcf's. Next, let us review some prior conjectures and results on the lsd and kcf's in the completely integrable case.

The problem of determining the lsd and kcf's of a spectrum was apparently raised by Wigner in his work on nuclear Hamiltonians, $H=1 / 24+V,([30,25])$. The potential $V$ was not known explicitly, so it made sense to consider it as somewhat random (see [14 or 25]). Wigner, therefore, proposed modeling the spectrum by the expected spectral properties of random $N \times N$ matrices in the limit $N \rightarrow \infty$. The spectral theory of random matrix ensembles was subsequently developed by Dyson, Mehta and others who in particular calculated explicitly the limiting lsd and kcf's for the main ensembles. At the same time, numerical studies of the spectrum of nuclear Hamiltonians, of Laplacians of various manifolds and domains, and of zeroes of the Riemann zeta function have produced a lot of data to compare with the random matrix lsd's and kcf's. These data seem to support 
Wigner's conjecture for Hamiltonians whose associated classical systems are in some sense chaotic $([25]$ p. $7,[2])$. However, the data go against the conjecture for simple systems like the Laplacian on a torus or the harmonic oscillator, which of course are completely integrable.

M.V. Berry and M. Tabor have proposed that the normalized eigenvalue spacings $S_{i}$ in the generic completely integrable case should behave like independent, Poisson distributed random variables, [3]. In particular, the spacings should be uncorrelated, and hence the spectrum should have more gaps and also more degeneracy than in the chaotic case. The heuristic idea seems to be this: As observed above, one approximates the eigenvalues by the values of the classical Hamiltonian $K=q \circ \chi$, expressed in terms of action-angle variables, at the lattice points $\mathbb{Z}^{n}+\mu$. Level spacings then correspond to values $S_{j}$ such that the region

$$
\left\{R \leqq K \leqq R+S_{j} / R^{n-1}\right\}
$$

contains a lattice point on each boundary component, but none in the interior ( $n$ is the dimension of $M$ ). In case $K$ is homogeneous, the surfaces $K=R$ are dilates of $K=1$, and the values $S_{j}$ may be viewed as the normalized spacings between the times when the dilating family $K=R$ touches a lattice point. If the surface $K=R$ is sufficiently "generic" (in some sense), these times are supposed to be Poisson, i.e. like the times at which a radioactive nucleus emits a particle ([3] p. 379).

A precise definition of "generic" has not been attempted in [3], but it certainly excludes systems, such as the harmonic oscillator, for which $K^{\prime \prime}=0$. In fact, the authors suggest that no unique lsd and kcf's exist in those examples, and in dimension two this has recently been proved by P. Bleher, [5]. The Zoll case we study is also non-generic in the sense that $K^{\prime \prime}=0[11]$, p. 32. However, it is completely unlike the case of harmonic oscillators in that unique 1sd's and kcf's do exit.

The heuristic principle we described above does not seem very plausible for individual integrable systems. For example, if $K=1$ is real analytic, then the times at which dilates touch lattice points along one direction would determine the times for any other direction. The spacings would then seem highly correlated and far from Poisson.

It would seem however to make more sense if the contour $K=1$ is itself regarded as random, and in particular if the law is such that modifications of $K=1$ at one point are independent of modifications at any other point. Such a random set up has recently been studied by Sinai, [27], and subsequently by Major, [24]. They consider the spectrum of the Laplacian on torii of revolution (obtained by revolving a symmetric profile curve $y=G(x),-1 \leqq x \leqq 1$ around the $x$ axis, and identifying the ends $x= \pm 1$ ). Such torii have completely integrable geodesic flows. Sinai defines a probability measure on the space of $G$ 's so that the resulting measure on the space of $K$ 's has the independence property alluded to above. He shows that the average value of the lsd for these $K$ exists and equals a generalized Poisson distribution. Major develops these ideas further to show that, with probability one, each curve $K$ has such an lsd. However, the $K$ in this set do not, unfortunately, seem to correspond to smooth metrics on torii of revolution, [24].

Finally, we note that in dimension two, completely integrable geodesic flows are only possible for metrics on the sphere and torus. In the case of the torus, the above metrics of revolution are apparently the only known ones. In the case of $S^{2}$, the only known completely integrable metrics are metrics of revolution and Zoll 
metrics. Thus, Zoll metrics actually form the most substantial family (of functional dimension two rather than one) of completely integrable surface metrics.

\section{Pair Correlation of a Zoll Metric}

3.1. Statement of the Result. Before stating our result, we need to introduce some notation. Let

$$
H: \mathcal{O} \rightarrow \mathbb{R}
$$

denote the function induced on $\mathcal{O}$ by the principal symbol of the operator $A$ of (2). Let $S^{*}\left(S^{2}\right)$ be the unit cosphere bundle of $S^{2}$. By definition of $\mathcal{O}$, we have a natural projection

$$
\pi: S^{*}\left(S^{2}\right) \rightarrow \mathcal{O}
$$

which is in fact a circle bundle, the action given by the Hamilton flow of $H_{0}(x, \xi)=|\xi|$ restricted to $S^{*}\left(S^{2}\right)$. Let $\alpha$ denote the pull-back to $S^{*}\left(S^{2}\right)$ of the canonical one-form on $T^{*} S^{2}$. Then it is trivial to check that $\alpha$ is a connection form on (8) whose curvature is the symplectic structure on $\mathcal{O}$.

Definition 3.1. For every closed curve $\gamma:[a, b] \rightarrow \mathcal{O}$, we will denote by $\mathfrak{h}(\gamma) \in$ $S^{1}=\mathbb{R} / 2 \pi \mathbb{Z}$ the holonomy of $\gamma$ with respect to the connection $\alpha$.

We will henceforth make the following

Assumption $\mathrm{M}: H: \mathcal{O} \rightarrow \mathbb{R}$ is a Morse function.

No explicit formula for $H$ is known in the case of Zoll surfaces. Hence one cannot check directly if the generic $H$ coming from a Zoll metric is Morse. However, our analysis certainly applies to generic zeroth-order perturbations of the round Laplacian, a class of operators very likely to include operators unitarily equivalent to generic Zoll Laplacians.

Let $\mathscr{R} \subset \mathbb{R}$ be the subset of the image of $H$ consisting of regular values, and let $c_{1}<\cdots<c_{K+1}$ be the critical values of $H(K \geqq 1)$. Then $\mathscr{R}$ is the disjoint union of $K$ open intervals, $\mathscr{R}_{v}$ :

$$
\mathscr{R}_{v}=\left(c_{v}, c_{v+1}\right), v \in\{1, \ldots, K\} .
$$

For every $v \in\{1, \ldots, K\}$ the inverse image $H^{-1}\left(\mathscr{R}_{v}\right)$ consists of a certain number $N(v)$ of connected components $X_{j}^{v}$, each one of which is diffeomorphic to a cylinder $S^{1} \times(0,1)$ :

$$
H^{-1}\left(\mathscr{R}_{v}\right)=\bigcup_{j=1}^{N(v)} X_{j}^{v}
$$

Definition 3.2. We will call $X_{j}^{v}$ the $(v, j)$ branch of $H$.

Each level surface $H^{-1}(E)$ with $E \in \mathscr{R}_{v}$ intersects each $X_{j}^{v}$ in a periodic trajectory $\gamma_{j}^{v}(E)$, whose minimal positive period will be denoted $T_{j}^{v}(E)$. As the notation suggests, for given $v$ and $j$ the period $T_{j}^{v}(E)$ can be regarded as a function of the energy $E$ in $\mathscr{R}_{v}$. Although not essential for the calculation of a pcf, we will make the following assumption for ease of exposition: 
Assumption T: For all $v, j$ the function $E \mapsto T_{j}^{v}(E)$ has non-zero derivative everywhere on $\mathscr{R}^{v}$.

On a branch one can also introduce global action-angle coordinates. Recall (see [1]) that the action coordinate is

$$
I_{j}^{v}=\frac{1}{2 \pi \sqrt{-1}} \log \mathfrak{h}\left(\gamma_{j}^{v}(E)\right),
$$

from which it follows that

$$
T_{j}^{v}(E)=\frac{d}{d E} \frac{1}{\sqrt{-1}} \log \mathfrak{h}\left(\gamma_{j}^{v}(E)\right) .
$$

By assumption $\mathrm{T}$, one can (and will) regard any of the functions $T_{j}^{v}, I_{j}^{v}$ and $H$ restricted to $X_{j}^{v}$ as a function of any of the other two.

Our result can be loosely stated as follows: the pair correlation function is determined by the pairs of (possibly identical) branches with the same index $v$ and commensurate period functions. Let's be more precise. For a given index $v$, let $S_{v}$ denote the set of all couples $(j, k), j, k \in\{1, \ldots, N(v)\}$ for which the functions $T_{j}^{v}$ and $T_{k}^{v}$ are related by an identity of the form

$$
T_{j}^{v}=\frac{p(j, k)}{q(j, k)} T_{k}^{v}
$$

with $p(j, k), q(j, k)$ relatively prime positive integers. The case $j=k$ and $p=1=q$ is not excluded; in fact the set $S$ is the graph of an equivalence relation in $\{1, \ldots, N(v)\}$. It follows from $(10)$ and $\forall(j, k) \in S_{v}$,

$$
\mathfrak{h}\left(\gamma_{j}^{v}(E)\right)^{q(j, k)} \mathfrak{h}\left(\gamma_{k}^{v}(E)\right)^{-p(j, k)}=e^{i c_{j, k}},
$$

for some constant $c_{j, k} \in \mathbb{R}$ and all $E \in \mathscr{R}_{v}$. With these notations we can now state our main result:

Theorem 3.3. Under assumptions $M$ and $T$, for every $\varphi$ with compactly supported Fourier transform one has

$$
\left(\rho_{2}^{l}, \varphi\right)=\frac{1}{2 \pi} \sum_{v=1}^{K} \sum_{(j, k) \in S_{v}} \sum_{r \in \mathbb{Z}} \frac{e^{i l r c_{j, k}} e^{i m_{j, k} r \pi / 4}}{p(j, k) q(j, k)^{2}} \int_{J(j, k ; v)} \hat{\varphi}(r t) t^{2}\left(\frac{d T_{j}^{v}}{d E}\right)^{-1}(t) d t
$$

modulo o(1), where $J(j, k ; v)$ is the interval

$$
J(j, k ; v)=\left[q(j, k) \min T_{j}^{v}, q(j, k) \max T_{j}^{v}\right]
$$

and $m_{j, k}$ is an integer (Maslov factor).

A couple of remarks. First, notice that since $\hat{\varphi}$ is compactly supported, the sum on the right-hand side is finite. Second, the apparent asymmetry of the $(j, k)$-th term evaporates if we recall (11). An equivalent, symmetrical formulation of the asymptotics in question is

$$
\frac{1}{2 \pi} \sum_{v=1}^{K} \sum_{(j, k) \in S_{v}} \sum_{r \in \mathbb{Z}} \frac{e^{i l r c_{j: k}} e^{i m_{j \cdot k} r \pi / 4}}{p(j, k) q(j, k)} \int_{R_{v}} \hat{\varphi}\left(r q T_{j}(E)\right) T_{j}(E) T_{k}(E) d E,
$$

which is symmetric in $(j, k)$ since $q T_{j}=p T_{k}$. 
3.2. Reduction to the Trace Formula. The proof of our result will be divided in two steps. The first reduces the problem to an application of the main result of [22] and the second is to carry out that calculation. Throughout we shall keep the previously introduced notation. Then

Let $P$ denote the operator on $S^{2}$ which on $H_{l}$ is multiplication by $l, \forall l \in \mathbb{Z}^{+}$.

$$
P=\sqrt{\Delta_{0}+1 / 4}-1 / 2,
$$

which by a theorem of Seeley implies that $P$ is a first-order pseudodifferential operator with symbol

$$
\sigma_{P}(x, \check{\xi})=H_{0}(x, \xi)=|\xi| .
$$

The unitary one-parameter group $\exp (i t P)$ is $2 \pi$ periodic, and the corresponding canonical transformations constitute an $S^{1}$ Hamiltonian action on $T^{*} S^{2} \backslash\{0\}$ which of course is just the geodesic flow parametrized by arc length.

Consider now on $S^{2} \times S^{2}$ the operators

$$
P_{1}=P \otimes I, \quad P_{2}=I \otimes P .
$$

These operators are commuting pseudodifferential operators with singular symbols, namely

$$
\sigma_{P_{1}}(x, \xi ; y, \eta)=|\xi|
$$

(and analogously for $\left.P_{2}\right)$, and away from the singular points $\left(\xi=0\right.$ for $P_{1}, \eta=0$ for $P_{2}$ ) their Hamilton vector fields are $2 \pi$-periodic. Hence they define a Hamiltonian action of the standard torus $T=S^{1} \times S^{1}$ on the symplectic manifold

$$
\check{X}=\left\{(x, \xi ; y, \eta) \in T^{*}\left(S^{2} \times S^{2}\right) ; \xi \neq 0 \text { and } \eta \neq 0\right\},
$$

with moment map

$$
\Phi: \check{X} \rightarrow \mathbb{R}^{2} \cong \mathrm{t}^{*}
$$

given by $\Phi(x, \xi ; y, \eta)=(|\xi|,|\eta|)$.

Our analysis will lead us to consider the following co-isotropic submanifold of $T^{*}\left(S^{2} \times S^{2}\right):$

$$
\Theta=\{(x, \xi ; y, \eta):|\xi|=|\eta|\} .
$$

The leaves of the null-foliation of this co-isotropic $\Theta$ are the fibers of the natural projection

$$
\Theta \rightarrow Y:=\Phi^{-1}\{(\lambda, \lambda) ; \lambda \in \mathbb{R}\} / K,
$$

where $K$ is the anti-diagonal subgroup

$$
K=\left\{\left(e^{i t}, e^{-i t}\right)\right\} \subset T .
$$

Lemma 3.4. $Y$ is a six-dimensional conic symplectic manifold with a free circular Hamiltonian action induced by the restriction of $\sigma_{P_{1}}$ to $\Theta$. Moreover, the symplectic quotient (reduction) of $Y$ with respect to this action is naturally symplectomorphic to the reduction of $\check{X}$ with respect to the $T$ action at $(1,1) \in \mathbb{R}^{2}$, which itself is symplectomorphic with $\mathcal{O} \times \mathcal{O}$ :

$$
\Phi^{-1}(1,1) / T=\mathcal{O} \times \mathcal{O} .
$$


This reduced symplectic manifold, $\mathcal{O} \times \mathcal{O}$, has a natural pre-quantum circle bundle, namely the quotient of $\Phi^{-1}(1,1)$ by $K$ :

$$
W=\left\{(x, \xi ; y, \eta) \in T^{*}\left(S^{2} \times S^{2}\right) ;|\xi|=1=|\eta|\right\} / K \rightarrow \mathcal{O} \times \mathcal{O} .
$$

In terms of the pre-quantum circle bundle $S^{*}\left(S^{2}\right) \rightarrow \mathcal{O}$ of $\mathcal{O}$, we can write (21) as

$$
W=\pi_{1}^{*} S^{*}\left(S^{2}\right) \otimes \pi_{2}^{*} S^{*}\left(S^{2}\right),
$$

where $\pi_{j}: \mathcal{O} \times \mathcal{O} \rightarrow \mathcal{O}$ is the projection onto the $j^{\text {th }}$ factor.

A simultaneous diagonalization of $P_{1}, P_{2}$ yields an orthogonal Hilbert space decomposition

$$
L^{2}\left(S^{2} \times S^{2}\right)=\bigoplus_{l, m} H_{l, m}
$$

where

$$
H_{l, m} \cong H_{l} \otimes H_{m}
$$

is the joint eigenspace of $P_{1}, P_{2}$ with joint eigenvalue $(l, m)$. Introduce the space

$$
\mathscr{H}=\bigoplus_{l} H_{l, l},
$$

and let $\Pi: L^{2}\left(S^{2} \times S^{2}\right) \rightarrow \mathscr{H}$ the orthogonal projector. This is the "ladder" of representations of $T$ associated to the line through the weight $(1,1)$.

Lemma 3.5. (See Theorem 6.7 of [20].) The projector $\Pi$ is a Fourier integral operator associated with the flow-out in $T^{*}\left(S^{2} \times S^{2}\right) \backslash\{0\}$ of the co-isotropic $\Theta$.

In [19], Guillemin and Sternberg show how the family of Fourier integral operators

$$
\mathscr{R}_{I I}=\{\Pi Q \Pi\},
$$

where $Q$ ranges over the pseudodifferential operators on $S^{2} \times S^{2}$ is a ring, which has a symbol calculus with phase space the reduced manifold $Y$. We will make use of this below. In fact, we will use a slight extension of it: we will consider Fourier integral operators of the form

$$
\Pi e^{i \theta P_{1}} Q \Pi \text {. }
$$

These are Fourier integral versions of the operators in $\mathscr{R}_{\Pi}$.

Consider next the operator

$$
A^{(2)}=A \otimes I-I \otimes A
$$

on $L^{2}\left(S^{2} \times S^{2}\right)$, where $A$ is the operator in (2). Clearly

$$
\left.\operatorname{Spec} A^{(2)}\right|_{H_{l, l}}=\left\{\mu_{l, j}-\mu_{l, k} ; j, k=1, \ldots, 2 l+1\right\} .
$$

Our main analytical object of study is the following:

$$
\Upsilon(\theta)=\operatorname{Tr}\left[\Pi \varphi\left(P_{1} A^{(2)}\right) e^{i \theta P_{1}} \Pi\right],
$$

where $\varphi$ is a function satisfying $\hat{\varphi} \in C_{0}^{\infty}(\mathbb{R}) . \Upsilon$ is a well-defined periodic distribution; in fact

$$
r(\theta)=\sum_{l=1}^{\infty} e^{i l \theta} \sum_{j, k=1}^{2 l+1} \varphi\left(l\left(\mu_{l, j}-\mu_{l, k}\right)\right) .
$$


We will next invoke Theorems 1.1 and 1.2 of [22], applied to the operator

$$
R=P_{1} A^{(2)}
$$

and the representation of $T$,

$$
\left(e^{i t_{1}}, e^{i t_{2}}\right) \mapsto e^{i\left(t_{1} P_{1}+t_{2} P_{2}\right)},
$$

both compressed by $\Pi$. By the remarks following Lemma 3.4, the symbolic calculation can be carried out in the reduced symplectic manifold $Y$. Two more technical remarks:

(1) The operators $R, P_{1}$ and $P_{2}$ have singular symbols. However, all the proofs and statements below occur microlocally in a neighborhood $\mathscr{U}$ of the inverse image by $\Phi$ of the diagonal, where all operators in sight have smooth symbols. Hence all the arguments go through in the present case.

(2) The operator $R$ is not elliptic in $\mathscr{U}$. One can easily check that what is needed in [22] is that $P$ be a first-order, self-adjoint pseudodifferential operator whose symbol does not vanish anywhere on $\Phi^{-1}(0)$. Since in our case $\Phi^{-1}(0)=\emptyset$ (i.e. the group action itself is elliptic) this is automatic.

Theorem 3.3 is proved by a symbolic calculation just like the one proving Theorem 1.2 of [22] (with the energy $E$ equal to zero). We will now indicate how the symbolic calculation goes, referring to [22] for more details. We break this discussion in the following two subsections.

3.3. Period Manifolds and Holonomies. The symbol of $R$ descends to a function $\rho$ on $\mathcal{O} \times \mathcal{O}$, which is simply

$$
\rho\left(z_{1}, z_{2}\right)=H\left(z_{1}\right)-H\left(z_{2}\right) .
$$

As in Theorem 1.1 of [22], the singularities of $Y$ are created by the periodic trajectories of $\rho$ on the energy surface $\{\rho=0\}$. More precisely, consider the pre-quantum circle bundle, $W$, of $\mathcal{O} \times \mathcal{O}$. Let us denote by $\mathfrak{h}(\gamma)$ the holonomy of a closed curve $\gamma \subset \mathcal{O} \times \mathcal{O}$ in this bundle.

Theorem 3.6. (See Theorem 1.1 of [22].) The singularities of $Y$ given by (29) are at those $\theta$ such that there exists a periodic trajectory of the Hamilton flow of $\rho, \gamma$, contained in $\rho^{-1}(0)$, and such that

$$
e^{i \theta}=\mathfrak{h}(\gamma) .
$$

Let us now study the space of periodic points $\left(z_{1}, z_{2}\right) \in \rho^{-1}(0)$. Consider the set $\mathscr{P}$ equal to

$$
\left\{\left(z_{1}, z_{2}, T\right) \in \mathcal{O} \times \mathcal{O} \times \operatorname{supp} \hat{\varphi} ; \rho\left(z_{1}, z_{2}\right)=0 \text { and }\left(z_{1}, z_{2}\right)=\exp \left(T \Xi_{p}\right)\left(z_{1}, z_{2}\right)\right\}^{1} \text {. }
$$

If $\left\{f_{t}\right\}$ denotes the Hamilton flow of $H$ on $\mathcal{O}$, then clearly

$$
\left(z_{1}, z_{2}, T\right) \in \mathscr{P} \Leftrightarrow H\left(z_{1}\right)=H\left(z_{2}\right) \quad \text { and } \quad z_{j}=f_{T}\left(z_{j}\right), \quad j=1,2 .
$$

It will suffice to work with the regular points in $\mathscr{P}$, that is, define for all $v \in\{1, \ldots, K\}$

$$
\mathscr{P}^{v}=\left\{\left(z_{1}, z_{2}, T\right) \in \mathscr{P} ; H\left(z_{j}\right) \in \mathscr{R}_{v}\right\},
$$

where, we recall, $\mathscr{R}_{v}$ denote the intervals of regular values of $H$.

\footnotetext{
${ }^{1} \Xi_{\rho}$ is the Hamilton vector field of $\rho$
} 
Lemma 3.7. Keeping the notation of Sect. 3.1, the connected components of $\mathscr{P}^{v}$ are the sets $\mathscr{P}_{j, k, r}^{v}$, where $(j, k) \in S_{v}$ and $r \in \mathbb{Z}$, consisting of all points $\left(z_{1}, z_{2}, T\right)$ with

$$
z_{1} \in X_{j}^{v}, \quad z_{2} \in X_{k}^{v}, \quad H\left(z_{1}\right)=H\left(z_{2}\right) \quad \text { and } \quad T=r q(j, k) T_{j}^{v}=r p(j, k) T_{k}^{v} .
$$

Notice that all these connected components have dimension three, whether $T=0$ or not. This will imply that all the singularities of $r$ have the same strength, and all will contribute to the pair correlation function.

Let us next compute the holonomy of a periodic trajectory of $\rho$ defined by a point in $\mathscr{P}_{j, k, r}^{v}$. (It is easy to see that all such trajectories have the same holonomy.) In general, the holonomy in $W$ of a curve on $\mathcal{O} \times \mathcal{O}$ is the product of the holonomies in $S^{*}\left(S^{2}\right)$ of the projections of the curve into the factors. Furthermore

$$
\exp \left(t \Xi_{\rho}\right)\left(z_{1}, z_{2}\right)=\left(f_{t}\left(z_{1}\right), f_{-t}\left(z_{2}\right)\right) .
$$

It follows that the holonomy in $W$ of the trajectories in $\mathscr{P}_{j, k, r}^{v}$ is

$$
e^{i r c_{j}, k}
$$

where the constants $c_{j, k}$ were defined in (12).

3.4. The Pair Correlation Density. Under certain "clean intersection" conditions we can symbolically obtain the asymptotic behavior of the Fourier coefficients of $Y$. The clean intersection condition is that $\mathscr{P}$ be the clean intersection of

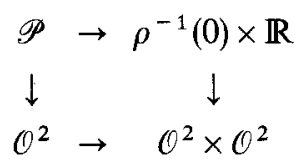

where $\mathcal{O}^{2}=\mathcal{O} \times \mathcal{O}$, the bottom arrow is the diagonal inclusion, and the right arrow is the Lagrangian immersion given by the flow of $\rho$. In the present case this is not a clean intersection diagram; however, it fails to be clean precisely at finitely-many points. Just as in [31], this is enough to yield the leading order behavior of the Fourier coefficients of $r$. The following is proved just like Theorem 1.2 and Corollary 7.2 of [23], working with $\Pi$-compressed operators and the phase space $Y$ :

Theorem 3.8. (See Theorem 1.2 and Corollary 7.2 of [23].) Under the assumptions $M$ and $T$, the clean intersection condition on (36) is satisfied in the complement of

$$
\bigcup_{\nu=1}^{K} \mathscr{P}^{v}
$$

for which furthermore $t$ is in the support of $\hat{\varphi}$. Moreover, the Fourier coefficients of $Y$ are asymptotic, as the frequency tends to infinity, to the sum over the connected components of $\mathscr{P}^{v}$ of

$$
e^{i r c_{j \cdot c}} \int_{\mathscr{P}_{j, k, r}} \hat{\varphi}(T) \delta,
$$

where $\delta$ is the density on $\mathscr{P}_{j, k, r}^{v}$ given by the product of the Liouville half-densities on $\mathcal{O}^{2}$ and on $\rho^{-1}(0) \times \mathbb{R}$.

We have observed already that all the connected components of $\mathscr{P}^{v}$ have the same dimension (three). For this reason each one contributes to the leading order behavior of the Fourier coefficients of $r$. 
We next determine the density $\delta$ appearing in (38). We present this calculation in the next subsection, in a slightly more general setting, for future reference.

3.4.1. The Trace Formula for Periodic Flows. We begin with some general considerations on the trace formula for periodic flows with variable period. Other than the case considered here, there are known examples of potentials in $\mathbb{R}^{n}$ having periodic energy levels, with variable period. Then the considerations below would apply to the semi-classical trace formula for them.

Let $(X, \omega)$ be a symplectic manifold, $G \in C^{\infty}(X)$ a Hamiltonian. Assume zero is a regular value of $G$, and that all trajectories of the Hamilton flow of $G$ on

$$
\Sigma=G^{-1}(0)
$$

are periodic, although NOT of the same period. For example, take $X=\mathscr{O} \times \mathcal{O}$ and $G=\rho$ as in the previous section; one knows there exist other examples of such Hamiltonians. More explicitly, we assume there is a smooth function,

$$
T: \Sigma \rightarrow \mathbb{R}^{+}
$$

such that

$\forall x \in \Sigma$ the trajectory through $x$ is periodic with minimal period $T(x)$.

Consider now the intersection diagram

$$
\begin{array}{ccc}
\mathscr{P} & \hookrightarrow & \sum \times \mathbb{R} \\
\downarrow & & \downarrow f \\
X & \rightarrow & X \times X,
\end{array}
$$

where the bottom arrow is the diagonal embedding, $f$ is the map

$$
f(x, t)=\left(x, \phi_{t}(x)\right),
$$

where $\left\{\phi_{t}\right\}$ is the Hamilton flow of $G$ restricted to $\Sigma$, and

$$
\mathscr{P}=\left\{(x, t) \in \mathbb{R} ; x=\phi_{t}(x)\right\} .
$$

Assuming that $\Sigma$ is connected, by (39), the decomposition of $\mathscr{P}$ into connected components takes the form

$$
\mathscr{P}=\bigcup_{k \in \mathbb{Z}} \mathscr{P}_{k}
$$

where

$$
\forall k \in \mathbb{Z} \quad \mathscr{P}_{k}=\{(x, k T(x)) ; x \in \Sigma\} .
$$

Obviously $\mathscr{P}$ is a smooth manifold, and each $\mathscr{P}_{k}$ is naturally diffeomorphic with $\Sigma$, being the graph of the function $k T$.

Lemma 3.9. Under the previous assumptions the diagram (40) is always clean.

Proof. Let $x \in \Sigma$ and $k \in \mathbb{Z}$. Assume $v \in T_{x} \Sigma$ and $\tau \in \mathbb{R}$ are such that

$$
v=d\left(\phi_{k T(x)}\right)(v)+\tau \Xi_{x},
$$


where $\Xi$ is the Hamilton vector field of $G$. This condition means that $d f_{(x, k T(x))}(v, \tau)$ is in the tangent space to the diagonal in $X \times X$. What we must prove is that this forces $(v, \tau)$ to be tangent to $\mathscr{P}_{k}$, which by (43) means that

$$
\tau=k d T_{x}(v) \text {. }
$$

Differentiating the identity

$$
\phi_{k T(x)}=x
$$

one sees that (44) is satisfied with any $v$ and $\tau=k d T_{x}(v)$. Hence (44) implies

$$
\tau \Xi_{x}=k d T_{x}(v) \Xi_{x} \text {. }
$$

Since zero is a regular value of $G, \Xi$ is nowhere zero on $\Sigma$ and this implies (45).

Endow $X$ with the half-density

$$
\delta_{X}=\frac{1}{\sqrt{n !}}\left|\omega^{n}\right|^{1 / 2},
$$

and $\Sigma \times \mathbb{R}$ with the half-density

$$
\delta_{\Sigma}=|d \lambda|^{1 / 2} \otimes|d t|^{1 / 2},
$$

where $d \lambda$ denotes Liouville density on $\Sigma$. One knows then that the clean intersection of $X$ and $\Sigma \times \mathbb{R}, \mathscr{P}$, inherits naturally a density $\delta$, which is roughly the product of $\delta_{X}$ and $\delta_{\Sigma}$ (see for example [22], Lemma 2.7). We now describe $\delta$.

Lemma 3.10. For every $k \in \mathbb{Z}$ identify $\mathscr{P}_{k}$ with $\Sigma$ in the natural way. Then the density $\delta$ on $\mathscr{P}_{k}$ is identified with Liouville measure, $|d \lambda|$.

Now in the trace formula, applied to a test function $\varphi$ with $\hat{\varphi} \in C_{0}^{\infty}$, one is to compute

$$
\int_{\mathscr{P}} \hat{\varphi}(t) \delta
$$

Clearly in the present case this integral equals

$$
\int_{\mathscr{T}} \hat{\varphi}(t) \delta=\sum_{k} \int_{\Sigma} \hat{\varphi}(k T(x)) d \lambda_{x} .
$$

Since $\hat{\varphi}$ is compactly supported, this sum is finite. The $k^{\text {th }}$ term in the sum can be written as the integral of $\hat{\varphi}$ against the push-forward of $d \lambda$ by the function $k T$ :

$$
\int_{\Sigma} \hat{\varphi}(k T(x)) d \lambda_{x}=\int_{\mathbb{R}} \hat{\varphi}(k t) d \mu_{t} \text {, where } d \mu=T_{*}(d \lambda) .
$$

We next compute $d \mu$ in the case of interest:

$$
X=\mathcal{O} \times \mathcal{O}, G=\rho .
$$

3.4.2. Computation of the pcf. We now go back to the computation of the pair correlation function, and apply the results of Sect. 3.3 to the calculation of Sect. 3.2.

Let $\left(I_{1}, \theta_{1}\right)$ and $\left(I_{2}, \theta_{2}\right)$ be two pairs of action-angle coordinates on a couple of branches $X_{j}^{v}, X_{k}^{v}$ with commensurate period, and let $T$ be the minimal common period function

$$
T_{j}^{v} q(j, k)=T=T_{k}^{v} p(j, k),
$$


see (11). Interpreted in the obvious way, this function is the primitive period function of $\rho\left(z_{1}, z_{2}\right)=H\left(z_{1}\right)-H\left(z_{2}\right)$ on

$$
\Sigma=\Sigma_{j, k}^{v}=\left(X_{j}^{v} \times X_{k}^{v}\right) \cap \rho^{-1}(0) .
$$

Lemma 3.11. Let $\imath$ : $\Sigma \hookrightarrow X$ be the inclusion map. Then Liouville measure on $\Sigma$ is defined by the three form

$$
\eta=\frac{1}{2 \pi} \frac{T}{p} \iota^{*}\left(d I_{1} \wedge d \theta_{1} \wedge d \theta_{2}\right)
$$

Proof. We begin with two identities: The symplectic form on $X=X_{j}^{v} \times X_{k}^{v}$ is

$$
\Omega=d I_{1} \wedge d \theta_{1}+d I_{2} \wedge d \theta_{2},
$$

which we write as

$$
\Omega=\frac{d I_{1}}{d H_{1}} d H_{1} \wedge d \theta_{1}+\frac{d I_{2}}{d H_{2}} d H_{2} \wedge d \theta_{2}
$$

where $H_{j}=H\left(z_{j}\right)$. Introduce $g=H_{1}+H_{2}$. Then, changing to the $\rho, g, \theta_{1}, \theta_{2}$ variables

$$
2 \Omega=d \rho \wedge\left(\frac{d I_{1}}{d H_{1}} d \theta_{1}-\frac{d I_{2}}{d H_{2}} d \theta_{2}\right)+d g \wedge\left(\frac{d I_{1}}{d H_{1}} d \theta_{1}+\frac{d I_{2}}{d H_{2}} d \theta_{2}\right)
$$

Hence:

$$
\begin{aligned}
\Omega^{2} & =\frac{1}{2} d \rho \wedge\left(\frac{d I_{1}}{d H_{1}} d \theta_{1}-\frac{d I_{2}}{d H_{2}} d \theta_{2}\right) \wedge d g \wedge\left(\frac{d I_{1}}{d H_{1}} d \theta_{1}+\frac{d I_{2}}{d H_{2}} d \theta_{2}\right) \\
& =\frac{d I_{1}}{d H_{1}} \frac{d I_{2}}{d H_{2}} d \rho \wedge d g \wedge d \theta_{1} \wedge d \theta_{2} .
\end{aligned}
$$

On the other hand,

$$
T_{j}^{v}=2 \pi \frac{d I_{1}}{d H_{1}}, \quad T_{k}^{v}=2 \pi \frac{d I_{2}}{d H_{2}},
$$

as functions on $X_{j}^{v}$, resp. $X_{k}^{v}$. We can then write

$$
\frac{\Omega^{2}}{2}=d \rho \wedge \eta_{0}
$$

with

$$
\eta_{0}=\frac{T_{j}^{\nu} T_{k}^{v}}{8 \pi^{2}} d g \wedge d \theta_{1} \wedge d \theta_{2}
$$

By definition, the pull-back of $\eta_{0}$ to $\Sigma$ is a three form defining the Liouville density of $\Sigma$. Since

and

$$
\imath^{*}(d g)=2 \imath^{*}\left(d H_{1}\right)=4 \pi \imath^{*}\left(\frac{1}{T_{j}^{v}} d I_{1}\right)
$$

$$
\iota^{*} T_{k}^{v}=\frac{T}{p},
$$

the lemma follows. 
Next we need to find a two-form, $\beta$, such that

From

$$
\eta=d T \wedge \beta \text {. }
$$

one easily gets

$$
T=q \imath^{*} T_{j}^{v}=2 \pi q \imath^{*} \frac{d I_{1}}{d H_{1}},
$$

$$
d T=\frac{4 \pi^{2} q^{2}}{T} \imath^{*}\left(\frac{d^{2} I_{1}}{d H_{1}} d I_{1}\right)
$$

and from here we find $\beta$ :

$$
\beta=\frac{T^{2}}{8 \pi^{2} p q^{2}} l^{*}\left(\left(\frac{d^{2} I_{1}}{d H_{1}^{2}}\right)^{-1} d \theta_{1} \wedge d \theta_{2}\right) .
$$

When we restrict $|\beta|$ to the fiber $T=t$ (which is the product of two circles) and integrate, we obtain the density of the pair correlation function:

$$
d \mu_{t}=\frac{1}{2 \pi p q^{2}} t^{2}\left(\frac{d^{2} I_{1}}{d H_{1}^{2}}\right)^{-1} d t .
$$

Here, as in Theorem 3.3, $H_{j}$ and $I_{j}$ are regarded as functions of the period, $t$. Applying Theorem 3.8 we obtain the formula appearing in Theorem 3.3.

\section{The Trace Formula for Commuting Operators}

In this section we sketch a proof of the semi-classical trace formula for commuting operators, which is needed for the calculation of the kcf. Since many of the results are simple extensions of the trace formula for a single operator we will be sketchy, and refer the reader to [22] for details.

4.1. Preliminary Results. Let $M$ be a compact manifold, and $Q_{1}, \ldots, Q_{m}$ be first order, self adjoint, commuting classical pseudodifferential operators on $M$. Let $W=T^{*} M \backslash\{0\}$, and denote the principal symbol of $Q_{j}$ by $q_{j}$.

Definition 4.1. We will say that $Q_{1}, \ldots, Q_{m}$ are a system of real principal type iff:

1. Zero is a regular value of the map

$$
\Phi=\left(q_{1}, \ldots, q_{m}\right): W \rightarrow \mathbb{R}^{m} .
$$

2. The radial vector field on $W$ is nowhere tangent to

$$
\Sigma=\Phi^{-1}(0) \text {. }
$$

The Hamilton vector fields associated with the principal symbols of the $Q_{j}$ are the infinitesimal generators of an $\mathbb{R}^{m}$ action on $W$. The result of acting by $t=\left(t_{1}, \ldots, t_{m}\right) \in \mathbb{R}^{m}$ on $w \in W$ will be denoted $f_{t}(w)$.

Lemma 4.2. Let $Q_{1}, \ldots, Q_{m}$ be a system of real principal type, and let $\varphi$ be a Schwartz function on $\mathbb{R}^{m}$ such that $\hat{\varphi} \in C_{0}^{\infty}\left(\mathbb{R}^{m}\right)$. Then the operator $\varphi\left(Q_{1}, \ldots, Q_{m}\right)$, defined by the spectral theorem, is a Fourier integral operator associated with the flow-out of $\Sigma$; more precisely with the canonical relation

$$
\mathscr{C}=\left\{\left(w, f_{t}(w)\right) ; t \in \operatorname{supp} \hat{\varphi} \text { and } w \in \Sigma\right\} .
$$


Proof. In an obvious sense,

$$
\varphi(Q)=\frac{1}{(2 \pi)^{m}} \int_{\mathbb{R}^{m}} e^{i t Q} \hat{\varphi}(t) d t .
$$

One can interpret this equation as follows. Let $\mathscr{E}$ denote the Schwartz kernel of $e^{i t Q}$ considered as an operator from $M$ to $M \times \mathbb{R}^{m}$. Thus $\mathscr{E}$ is a distribution on $M \times M \times \mathbb{R}^{m}$. Then (50) says that the Schwartz kernel of $\varphi(Q)$ is the push-forward of the product $\hat{\varphi}(t) \mathscr{E}$ by the natural projection

$$
\Pi: M \times M \times \mathbb{R}^{m} \rightarrow M \times M .
$$

One can easily prove (see e.g. [22]) that $\mathscr{E}$ is a Lagrangian distribution associated with the so-called character Lagrangian of $f$, which is

$$
\Gamma_{f}=\left\{\left(w, f_{t}(w)^{\prime}, t, \Phi(w)\right) ; w \in W, t \in \mathbb{R}^{m}\right\} .
$$

Since $\hat{\varphi}$ has compact support, the product $\hat{\varphi}(t) \mathscr{E}$ has compact support and the push-forward by $\Pi$ will be a Lagrangian distribution associated with $\mathscr{C}$ (49) if $\Gamma_{f}$ intersects cleanly the submanifold of $W \times W \times T^{*} \mathbb{R}^{m}$ given by

$$
\left\{\left(w, w^{\prime}, t, 0\right)\right\} \text {. }
$$

This is guaranteed by the first condition of $Q$ being of real principal type. The result now follows from a general theorem on the push-forward of Lagrangian distributions (see for example Chapter 6 of [21]).

Now assume that there is an additional first-order self adjoint classical pseudodifferential operator, $P$, commuting with the $Q_{j}$ and with the property

$$
\operatorname{spec}(P) \subset \mathbb{Z}
$$

Then $\theta \mapsto e^{i \theta P}$ is a representation of $S^{1}=\mathbb{R} / 2 \pi \mathbb{Z}$ by Fourier integral operators. For every $l \in \mathbb{Z}$, let $\mathscr{H}_{l}$ be the eigenspace of $P$ with eigenvalue $l\left(\mathscr{H}_{l}\right.$ may be trivial for some $l$ ). Then one has a Hilbert space direct sum,

$$
L^{2}(M)=\bigoplus_{l} \mathscr{H}_{l}
$$

Since the $Q_{j}$ commute with $P$, they preserve this decomposition. For each $l \in \mathbb{Z}$, let

$$
\vec{\lambda}_{l, j}=\left(\lambda_{l, j}^{1}, \ldots, \lambda_{l, j}^{m}\right), \quad j=1,2, \ldots
$$

be the joint spectrum of the operators $\left(Q_{1}, \ldots, Q_{m}\right)$ restricted to $\mathscr{H}_{l}$.

We will henceforth assume that $P, Q_{1}, \ldots, Q_{m}$ are jointly elliptic, that is

$$
p^{-1}(0) \cap \Sigma=\emptyset,
$$

where $p$ is the principal symbol of $P$. We are interested in the large $l$ asymptotics of

$$
\sum_{j} \varphi\left(\vec{\lambda}_{l, j}\right)
$$

The assumption of joint ellipticity ensures that, for each $l$, this series converges absolutely; in particular the exact ordering of the $\vec{\lambda}$ is irrelevant. 
4.2. Reduction. We will obtain the asymptotic behavior of (54), under suitable assumptions, but we will first need to do a little symplectic geometry. We begin by assuming that the Hamilton flow of $p$ induces a free action of $S^{1}$ on $Z=p^{-1}(1)$; then the quotient space

$$
X:=Z / S^{1}
$$

is a manifold with a natural symplectic structure. More precisely, if $\alpha_{W}$ is the canonical one-form on $W$ and $t: Z \subseteq W$ is the inclusion,

$$
\alpha:=\imath^{*} \alpha_{W}
$$

is a connection form on the circle bundle

$$
\pi: Z \rightarrow X
$$

whose curvature is $(-1)$ times the natural symplectic structure of $X$.

The functions $q_{j}$ Poisson commute with $p$, and hence there are smooth functions $H_{j} \in C^{\infty}(X)$ such that

$$
\left.q_{j}\right|_{Z}=\pi^{*}\left(H_{j}\right) .
$$

The $H_{j}$ Poisson commute among themselves in $X$, and thus define a Hamiltonian $\mathbb{R}^{m}$ action on $X$, which we denote by $\phi_{t}$. Our next assumption is: zero is a regular value of the map

$$
\Psi=\left(H_{1}, \ldots, H_{m}\right): X \rightarrow \mathbb{R}^{m} .
$$

By Lagrange multipliers this is equivalent to say that $(0, \ldots, 0,1)$ is a regular value of the map $\left(q_{1}, \ldots, q_{m}, p\right)$.

The previous assumption implies that $X_{0}=\Psi^{-1}(0)$ is a codimension $m$ submanifold of $X$. It is known that the flow out of $\phi$ is a canonical relation on $X$; consider the diagram

$$
\begin{array}{ccc}
\mathscr{P} & \rightarrow \Psi^{-1}(0) \times U \\
\downarrow & \downarrow \phi \\
X \rightarrow & X \times X,
\end{array}
$$

where $U$ is an open set containing the support of $\hat{\varphi}$, and $\mathscr{P}$ is the fiber product:

$$
\mathscr{P}=\left\{(x, T) \in X_{0} \times U ; x=\phi_{T}(x)\right\} .
$$

Our final assumption is that this is a clean diagram, meaning that $\mathscr{P}$ is a manifold and the tangent space of $\mathscr{P}$ at any point $(x, T)$ is the intersection of the image of $d \phi$ at $(x, T)$ with the diagonal.

If $\phi^{j}$ denotes the Hamilton flow of $H_{j}$, then

$$
\phi_{T}=\phi_{T_{1}}^{1} \circ \cdots \circ \phi_{T_{m}}^{m},
$$

and so $\mathscr{P}$ consists in, loosely speaking, those points in $X_{0}$ which are periodic under a combination of the flows $\phi^{j}$. Let's be more precise. Assume $x \in X_{0}$ satisfies

$$
\phi_{T_{1}}^{1} \cdots \circ \phi_{T_{m}}^{m}(x)=x .
$$

Then the $\phi$-orbit through $x$ is covered by

$$
\mathbb{R}^{m} / T \mathbb{Z}^{m}:=\mathbb{R}^{m} / T_{1} \mathbb{Z} \times \cdots \times T_{m} \mathbb{Z} .
$$


If the covering is trivial we will say that $T$ is the primitive period of $x$. More precisely, the covering is given by the flow evaluated at $x$ : consider the map

$$
\begin{aligned}
& g: \mathbb{R}^{m} / T \mathbb{Z}^{m} \rightarrow X \\
& \left(t_{1}, \ldots, t_{m}\right) \mapsto \phi_{t_{1}}^{1} \circ \cdots \circ \phi_{t_{m}}^{m}(x) .
\end{aligned}
$$

The following is easy to prove:

Lemma 4.3. The map $g$ is a covering map of the orbit of $x$ which is an isotropic submanifold, that is, if $\Omega$ is the symplectic form of $X$,

$$
g^{*} \Omega=0 .
$$

It follows that the pull-back, $g^{*} Z$, of the circle bundle $Z$ with its induced connection is a flat bundle. Hence if $\gamma$ is a closed curve in $\mathbb{R}^{m} / T \mathbb{Z}^{m}$, the holonomy with respect to $\alpha$ of the curve $g \circ \gamma$ only depends on the homology class of $\gamma$. An important number attached to the couple $(x, t)$ is the holonomy of the "diagonal" homology class in $\mathbb{R}^{m} / T \mathbb{Z}^{m}$, represented for example by the closed curve

$$
\begin{aligned}
{[0,1] } & \rightarrow X \\
s & \mapsto \phi_{s T_{1}}^{1} \circ \cdots \circ \phi_{s T_{m}}^{m}(x) .
\end{aligned}
$$

We will denote the holonomy of this curve by $\mathfrak{h}(x, T)$.

Corollary 4.4. If the diagram (55) is clean and $K \subset \mathbb{R}$ is compact, the set

$$
\{\mathfrak{h}(x, T) ;(x, T) \in \mathscr{P}, T \in K\} \subset S^{1}
$$

is finite.

Proof. The map $\mathfrak{h}: \mathscr{P} \rightarrow S^{1}$ is locally constant, by the previous lemma.

4.3. Conclusions. We are now ready to state the trace formula, and to sketch some applications.

Theorem 4.5. If the diagram (55) is clean, the distribution

$$
r(s)=\sum_{l=1}^{\infty} \sum_{j} \varphi\left(\vec{\lambda}_{l, j}\right) e^{i s l}
$$

is a classical Lagrangian distribution on $S^{1}$ with (finitely-many) singularities at the points $\mathfrak{h}(x, T)$ with $(x, T) \in \mathscr{P}$.

Proof. We sketch the proof. One first notices that

$$
\Upsilon(s)=\operatorname{Tr}\left(\Pi \varphi\left(Q_{1}, \ldots, Q_{m}\right) e^{i s P}\right),
$$

where $\Pi$ is the Szegö projector. The punctured cotangent space $T^{*} S^{1} \backslash\{0\}$ of $S^{1}$ has two components, $T^{+}$and $T^{-}$. Symbolically, the action of $\Pi$ on a Lagrangian distribution is to "erase" the part of the wave-front set contained in $T^{-}$, leaving untouched (symbol and all) the part contained in $T^{+}$. In other words, it is enough to show that $\operatorname{Tr} \varphi\left(Q_{1}, \ldots, Q_{m}\right) e^{i s P}$ is a Lagrangian distribution and compute its symbol. 
The process of forming the trace of $\varphi\left(Q_{1}, \ldots, Q_{m}\right) e^{i s P}$ is the application of a certain Fourier integral operator to the Schwartz kernel of

$$
\varphi\left(Q_{1}, \ldots, Q_{m}\right) e^{i s P} .
$$

The latter is a Lagrangian distribution, so the trace will likewise be Lagrangian provided a certain "clean intersection" condition is satisfied. Exactly as in [22], that condition can be shown to be equivalent to the cleanness of the diagram (55).

It remains to be shown that the singularities of $Y$ are at the holonomies of periodic orbits. By Lemma 4.2, the singularities of the trace of (58) (as a distribution in $s$ ) are easily seen to be at those $s$ for which there is a point $w \in \Sigma$ and a $t$ in the support of $\tilde{\varphi}$ such that

$$
e^{i s} \cdot f_{t}(w)=w
$$

where we have denoted with a dot the action of the Hamilton flow of $p$. Since $p$ Poisson commutes with the $q_{j}$ 's (and by the joint ellipticity), there is an $w$ satisfying this equation iff there is an $w$ satisfying this and, additionally, $p(w)=1$. Such an $w$ projects to a periodic trajectory of $\phi$, of period $t$. Hence all we must show is that the map

$$
\begin{aligned}
\mathbb{R}^{m} & \rightarrow \Sigma \\
t & \mapsto f_{t}(w)
\end{aligned}
$$

is horizontal with respect to $\alpha$. The image of $\partial / \partial t_{j}$ under the differential of this map is the Hamilton vector field of $q_{j}$ at points on $\Sigma$. Since $q_{j}$ is homogeneous of degree one, the contraction of $\alpha_{W}$ and the Hamilton vector field of $q_{j}$ is equal to $q_{j}$, which is identically zero on $\Sigma$. Hence for every $j$ the image of $\partial / \partial t_{j}$ is hórizontal.

The principal symbols $a_{0}^{r}$ of the singularities of $r$ at the $s_{r}$ determine the leading order asymptotics of the Fourier coefficients of $r$.

Theorem 4.6. If the diagram (55) is clean, there is an asymptotic expansion of the Fourier coefficients of $r$ of the following form: As $l \rightarrow \infty$,

$$
\sum_{j} \varphi\left(\vec{\lambda}_{l, j}\right) \sim \sum_{r=1}^{N} e^{i l s_{r}} \sum_{k=0}^{\infty} a_{k}^{r} l^{d_{r}-k},
$$

where:

(i) The index $r$ labels the connected components $\mathscr{P}_{r}$ of $\mathscr{P}$ with periods in the support of $\hat{\varphi}$,

(ii) For each $r, e^{i s_{r}}$ is the holonomy of the corresponding trajectory,

(iii) For each $r$

$$
a_{0}^{r}=e^{i \mu_{r} \pi / 4} \int_{\mathscr{P}_{r}} \hat{\varphi}(t) \delta_{r},
$$

where $\delta_{r}$ is the density on $\mathscr{P}_{r}$ product of the Liouville densities of the diagonal in $X \times X$ and the flow-out of $X_{0}$ and $\mu_{r}$ is an integer (Maslov factor). 


\section{5. $k$-level Correlation Functions}

In this section we describe a formula for the kcf and sketch its proof.

5.1. Statement of the Formula. Recall Definition 1.3:

$$
\rho_{k}^{l}=\frac{1}{2 l+1} \sum_{i_{1}, \ldots, i_{k}} \delta_{l\left(\mu_{l}, i_{2}-\mu_{l}, i_{1}, \ldots, \mu_{l}, i_{k}-\mu_{l}, i_{k-1}\right)} .
$$

We will reduce the computation of the weak limit of these distributions as $l \rightarrow \infty$ to the trace formula for $k-1$ commuting operators with a circular symmetry having for reduced phase space the product of $k$ copies of $\mathcal{O}$ :

$$
X=\mathcal{O} \times \cdots \times \mathcal{O}
$$

( $k$ factors). We next describe the formula for the kcf, which is a natural generalization of the pair-correlation formula found in Sect. 3 .

We keep the notation of Sect. 3.1: $H: \mathcal{O} \rightarrow \mathbb{R}$ is the reduced symbol of the operator $A$. We will assume it is a Morse function. Denoting points in $X$ by $z=\left(z_{1}, \ldots, z_{k}\right), z_{j} \in \mathcal{O}$, define

$$
H_{j}(z)=H\left(z_{j}\right), \quad 1 \leqq j \leqq k
$$

and

$$
G_{j}=H_{j}-H_{j+1}, \quad 1 \leqq j \leqq k-1 .
$$

Then $G_{1}, \ldots, G_{k-1}$ are commuting Hamiltonians on $X$.

We will prove that the $\mathrm{kcf}$ is a sum over $k$-tuples of branches of $H$ with the same index $v$ and rationally related period functions. For simplicity of notation we will simply state the summand corresponding to a $k$-tuple

$$
X_{i_{1}}^{v}, \ldots, X_{i_{k}}^{v},
$$

where the same branch may appear repeatedly with different subindices. Let $T_{j} \in C^{\infty}\left(\mathscr{R}_{v}\right)$ be the period function of $X_{i_{j}}^{v}, 1 \leqq j \leqq k$. The assumption that the period functions are rationally related is that there exist integers $m_{h}$ such that

$$
m_{1} T_{1}+\cdots+m_{k} T_{k}=0 .
$$

This implies that every point in $X_{i_{1}}^{v} \times X_{i_{k}}^{v}$ is periodic for the $\mathbb{R}^{k-1}$ action generated by $\left(G_{1}, \ldots, G_{k-1}\right)$. Let's denote by $e^{i s}$ the holonomy of this periodic manifold, in the sense of Corollary 4.4 (see also Lemma 5.4).

Finally, let $\left(I_{j}, \theta_{j}\right)$ be action-angle coordinates on $X_{j}^{v}$. We will regard the period functions $T_{j}$ as functions of a real variable $h$, namely the Hamiltonian. We can now state the formula for the $\mathrm{kcf}$ :

Theorem 5.1. Under the same assumptions as in Theorem 3.3, the sequence $\left\{\rho_{k}^{l}, l=1,2, \ldots\right\}$ has a unique weak limit $\rho_{k}$. Moreover, if $\varphi$ is a test function with $\hat{\varphi} \in C_{0}^{\infty}\left(\mathbb{R}^{k}\right),\left(\rho_{k}^{l}, \varphi\right)$ is asymptotic, as $l \rightarrow \infty$, to the sum over all $k$-tuples (64) satisfying (65), of the following terms:

$$
C e^{\pi i m / 4} e^{i l s} \int_{\mathscr{R} v} \hat{\varphi}\left(F_{1}(h), \ldots, F_{k-1}(h)\right)\left(\prod_{j=1}^{k} T_{j}(h)\right) d h,
$$


where $m$ is an integer (Maslov factor) depending on the $k$-tuple, $C$ a universal constant and

$$
F_{j}(h)=m_{1} T_{1}(h)+\cdots+m_{j} T_{j}(h) .
$$

5.2. Reduction to the Trace Formula. We begin the proof by showing how the kcf for a Zoll metric can be computed by an application of the trace formula for $k-1$ commuting operators. We will work on

$$
M=S^{2} \times \cdots \times S^{2} \quad(k \text { factors }) .
$$

We will consider the following operators acting on functions on $M$ :

$$
A_{j}=I \otimes \cdots A \otimes \cdots I, \quad 1 \leqq j \leqq k,
$$

where $A$ is the operator in (2) and it appears in the $j^{\text {th }}$ position. We then define, for $1 \leqq j \leqq k-1$, the operators

$$
Q_{j}=A_{j}-A_{j+1} .
$$

We are actually interested in the compression of the $Q_{j}$ to the "diagonal" ladder, which we now define. Clearly

$$
L^{2}(M)=\widehat{\bigoplus}_{i} \mathscr{H}_{i_{1}}^{1} \otimes \cdots \otimes \mathscr{H}_{i_{k}}^{k},
$$

where $\mathscr{H}_{i_{j}}^{j}$ is the space of spherical harmonics of degree $i_{j}$ considered as functions of the $j^{\text {th }}$ component of $M$. Define

$$
\mathscr{H}=\bigoplus_{l=1}^{\infty} \mathscr{H}_{l}^{1} \otimes \cdots \otimes \mathscr{H}_{l}^{k},
$$

and

$$
\Pi: L^{2}(M) \rightarrow \mathscr{H}
$$

the orthogonal projection.

Lemma 5.2. The projector $\Pi$ is a Fourier integral operator whose canonical relation is the flow-out of the following coisotropic in $T^{*} M \backslash\{0\}$ :

$$
\Theta=\left\{\left(x_{1}, \xi_{1}, \ldots, x_{k}, \xi_{k}\right) ; \forall i, j\left\|\xi_{i}\right\|=\left\|\xi_{j}\right\|\right\} .
$$

Proof. On $M$ we have $k$ commuting operators which generate a unitary representation of the circle group, namely

$$
P_{j}=I \otimes \cdots P \otimes \cdots I \quad 1 \leqq j \leqq k,
$$

where $P$ is the operator (14) in the $j^{\text {th }}$ factor. For our purposes, $P_{j}$ can be treated as a standard first-order pseudodifferential operator with symbol

$$
p_{j}\left(x_{1}, \xi_{1}, \ldots, x_{k}, \xi_{k}\right)=\left\|\xi_{j}\right\| .
$$

The space $\mathscr{H}$ is the space of vectors transforming under this representation by one of the irreducible representations with weight of the form $(l, \ldots, l), l>0$. Notice that $\Theta$ is the inverse image of the ray $\{(\lambda, \ldots, \lambda) ; \lambda \in \mathbb{R}) \subset \mathbb{R}^{k}$ under the moment map of the torus action, namely $\left(p_{1} \ldots p_{k}\right)$. Now the proof follows that of Theorem 6.7 of $[20]$. 
The coisotropic $\Theta$ is of course conic. It fibrates over a conic symplectic manifold, $W$, of dimension $2 k+2$. This symplectic manifold is the phase space of the algebra of Toeplitz-type operators of the form

$$
\Pi \circ Q \circ \Pi \text {, }
$$

where $Q$ ranges over the pseudodifferential operators on $M$, see [19]. We are particularly interested in the operators

$$
Q_{j}^{\Pi}=\Pi \circ Q_{j} \circ \Pi
$$

and

$$
P^{\Pi}=\Pi \circ P_{j} \circ \Pi
$$

(by the definitions the latter is the same operator for all $j$ ). We summarize some rather obvious facts about these operators:

Lemma 5.3. The non-zero eigenvalues of $P^{I}$ are the positive integers, and the eigenspace of $l$ is $\mathscr{H}_{l}^{1} \otimes \cdots \otimes \mathscr{H}_{l}^{k}$. Furthermore, the operators $Q_{j}^{\Pi}$ commute among themselves and with $P^{I I}$, and the joint spectrum of the restriction of $\left(Q_{1}^{I}, \ldots, Q_{k-1}^{I}\right)$ to $\mathscr{H}_{l}^{1} \otimes \cdots \otimes \mathscr{H}_{l}^{k}$ consists of

$$
\vec{\mu}_{l, i}=\left(\mu_{l, i_{1}}-\mu_{l, i_{2}}, \ldots, \mu_{l, i_{k-1}}-\mu_{l, i_{k}}\right),
$$

where $i$ is a multi-index with $1 \leqq i_{j} \leqq 2 l+1$.

The kcf is the weak limit of $\frac{1}{2 l+1}$ times the distribution of the $\vec{\mu}_{l, i}$ as $l \rightarrow \infty$. But this is precisely what the trace formula for $\left(Q_{1}^{I}, \ldots, Q_{k-1}^{\Pi}\right)$ computes, under the circular symmetry generated by $P^{\Pi}$. Although these operators are not pseudodifferential, by the symbol calculus for Toeplitz-type operators associated with fibrating coisotropics, developed in [19], the statements of the previous sections remain valid, provided we use the symplectic manifold $W$ as the initial phase space of the problem. We now make that calculation explicit.

5.3. The End of the Proof. As stated, the co-isotropic submanifold $\Theta$ fibrates over a symplectic manifold $W$ of dimension $2 k+2$. To see this, notice that the nullleaves of $\Theta$ are the orbits of a free Hamilonian action of the $(k-1)$-dimensional torus, generated by the $k-1$ Hamiltonians

$$
\left(x_{1}, \xi_{1}, \ldots, x_{k}, \xi_{k}\right) \mapsto\left\|\xi_{j}\right\|-\left\|\xi_{j+1}\right\|, \quad 1 \leqq j \leqq k-1 .
$$

Then $W$ is the quotient of $\Theta$ by this action. Let

$$
\rho: \Theta \rightarrow W
$$

be the natural projection, and denote by $p: W \rightarrow \mathbb{R}$ the function induced by the restriction to $\Theta$ of the principal symbol of $P_{1}$, namely

$$
\begin{aligned}
\Theta & \rightarrow \mathbb{R} \\
\left(x_{1}, \xi_{1}, \ldots, x_{k}, \xi_{k}\right) & \mapsto\left\|\xi_{j}\right\|
\end{aligned}
$$

(any $j$ ). The Hamilton flow of $p$ induces a free action of $S^{1}=\mathbb{R} / 2 \pi \mathbb{Z}$ on $W$. The trace formula of the previous section involves the reduction of $W$ with respect to this action of the circle. 
Lemma 5.4. The reduction of $W$ with respect to $p$, that is

$$
X:=p^{-1}(1) / S^{1}
$$

is, as a symplectic manifold, isomorphic with the product of $k$ copies of the manifold of oriented great circles, $\mathcal{O}$ :

$$
X=\mathcal{O} \times \cdots \times \mathcal{O}
$$

Proof. Since one can "reduce in stages," $X$ can be identified with the space of leaves of the null foliation of the coisotropic

$$
\Theta_{1}:=\Theta \cap\left\{\left\|\xi_{1}\right\|=1\right\}=\left\{\left(x_{1}, \xi_{1}, \ldots, x_{k}, \xi_{k}\right) ; \forall i\left\|\xi_{i}\right\|=1\right\} .
$$

This is precisely the Cartesian product of $k$ copies of the unit cosphere bundle of $S^{2}$. Since $\mathcal{O}$ is the space of null leaves of the latter, we are done.

Next we identify the reduced flow of the symbols of the operators $Q_{j}$. Recall the notation $(62,63)$ and let $\pi: \Theta_{1} \rightarrow X$ be the natural projection. It is obvious that $\pi^{*} H_{j}$ is the restriction to $\Theta_{1}$ of the principal symbol of $A_{j}$, while $\pi^{*} G_{j}$ is the restriction of the principal symbol of $Q_{j}$ to $\Theta_{1}$. Hence $G_{1}, \ldots, G_{k-1}$ are the reduced symbols of the Toeplitz-type operators $Q_{j}^{I}$. Let

$$
\begin{aligned}
\Psi: X & \rightarrow \mathbb{R}^{k-1} \\
z & \mapsto\left(G_{1}(z), \ldots G_{k-1}(z)\right)
\end{aligned}
$$

be the the moment map of the resulting $\mathbb{R}^{k-1}$ action. As we know now from the previous section, the kcf is determined by the points in $\Psi^{-1}(0)$ which are mapped onto themselves by the action of some $t \in \mathbb{R}^{k-1}$ with $t$ in the support of the Fourier transform of the test function appearing in the kcf. We now discuss this $\mathbb{R}^{k-1}$ action and the Liouville measure of its period manifold.

It is clear that, if $\left\{f_{s}\right\}$ denotes the Hamilton flow of $H$ on $\mathcal{O}$, the Hamilton flow of $G_{j}$ is

$$
(s, z) \mapsto\left(z_{1}, \ldots, f_{s}\left(z_{j}\right), f_{-s}\left(z_{j+1}\right), \ldots, z_{k}\right) .
$$

Hence the action of $t=\left(t_{1}, \ldots, t_{k-1}\right) \in \mathbb{R}^{k-1}$ is

$$
\psi_{t}(z)=\left(f_{t_{1}}\left(z_{1}\right), \ldots, f_{t_{j}-t_{j-1}}\left(z_{j}\right), \ldots, f_{-t_{k-1}}\left(z_{k}\right)\right),
$$

and periodicity of $z$ with period $t$ is equivalent to the equations

$$
f_{s_{j}}\left(z_{j}\right)=z_{j}, \quad 1 \leqq j \leqq k,
$$

where

$$
s_{1}=t_{1}, \quad s_{k}=-t_{k-1} \quad \text { and } \quad \forall 2 \leqq j \leqq k-1, \quad s_{j}=t_{j}-t_{j-1} .
$$

Conversely, $\psi$-periodic orbits are solutions of (68) under the additional condition that

$$
s_{1}+s_{2}+\cdots+s_{k}=0,
$$

which means that the $s_{j}$ are of the form (69). In addition, to contribute to the kcf the point $z$ must satisfy $z \in \Psi^{-1}(0)$, which is equivalent to

$$
H\left(z_{1}\right)=H\left(z_{2}\right)=\cdots=H\left(z_{k}\right) .
$$


It follows from (68) to (71) that the $\mathrm{kcf}$ is determined by the $k$-tuples of branches having rationally related primitive period functions and same index $\nu$. Specifically, if $X_{j_{1}}^{\nu}, \ldots, X_{j_{k}}^{v}$ is a $k$-tuple of branches whose primitive period functions satisfy a relation of the form $(65)$, namely

$$
m_{1} T_{1}+\cdots+m_{k} T_{k}=0,
$$

where the $m_{j}$ 's are integers, then we obtain a solution of (68) and (70) by letting

$$
\left(z_{1}, \ldots, z_{k}\right) \in X_{1}^{v} \times \cdots \times X_{k}^{v} \text { and } \forall j s_{j}=m_{j} T_{j}\left(z_{j}\right) .
$$

Just as in the case $k=2$, the diagram (55) is clean off a submanifold of positive codimension, and the data above determine a connected component of $\mathscr{P}$ in the region of clean intersection. The periods $t$ of $\psi$ are, inverting the upper triangular transformation (69),

$$
t_{j}=m_{1} T_{1}+m_{2} T_{2}+\cdots+m_{j} T_{j}
$$

To be more precise, let

$$
Y:=\Psi^{-1}(0) \cap\left(X_{1}^{v} \times \cdots \times X_{k}^{v}\right) .
$$

Every point in $Y$ is $\psi$-periodic: define the period map $\mathscr{T}$ by

$$
\begin{aligned}
\mathscr{T}: Y & \rightarrow \mathbb{R}^{k-1} \\
z & \mapsto\left(t_{1}(z), \ldots, t_{k-1}(z)\right),
\end{aligned}
$$

where the $t_{j}$ are given by (72) $\left(T_{j}\right.$ is regarded as a function of $\left.z_{j}\right)$. Clearly

$$
\forall z \in Y \quad \psi_{\mathscr{T}(z)}(z)=z
$$

Then the graph of $\mathscr{T}$

$$
\mathscr{P}_{0}=\{(z, t) ; z \in Y, t=\mathscr{T}(z)\}
$$

is an open set of $\mathscr{P}$ where the intersection is clean. The general clean connected component of $\mathscr{P}$ is of this form if we do not assume that the $m$ 's are relatively prime.

Lemma 5.5. Let $\pi: \mathscr{P}_{0} \rightarrow \mathbb{R}^{k-1}$ be the natural projection. Just as in the trace formula for a single operator with periodic null-bicharacteristics, the integral of $\pi^{*} \hat{\varphi}$ with respect to the density induced by the Liouville densities in the diagram (55) is equal to

$$
\int_{Y}\left(\mathscr{T}^{*} \hat{\varphi}\right) \eta
$$

where $\eta$ is the Liouville form on $Y$.

Proof. This is trivially equivalent to the statement that under the obvious diffeomorphism between $\mathscr{P}_{0}$ and $Y$ the density on $\mathscr{P}_{0}$ corresponds to Liouville measure on $Y$. We leave the proof of this as an exercise.

We will compute the Liouville measure on $Y$ in a moment, but first some remarks on the function $\mathscr{T}$. Introduce action-angle coordinates $I_{j}, \theta_{j}$ on the $j^{\text {th }}$ branch, $1 \leqq j \leqq k$. Then $T_{j}$ is a function of $H_{j}$, namely

$$
T_{j}=2 \pi \frac{d I_{j}}{d H_{j}} .
$$


It follows that there exist functions $F_{j}$ of a real variable, $\forall 1 \leqq j \leqq k-1$, such that

$$
\mathscr{T}\left(z_{1}, \ldots, z_{k}\right)=\left(F_{1} \circ H, \ldots, F_{k-1} \circ H\right),
$$

where $H$ denotes the function on $Y$ such that

$$
H\left(z_{1}, \ldots, z_{k}\right)=H_{j}\left(z_{j}\right)
$$

for any $j$. In fact,

$$
F_{j}=m_{1} T_{1}+\cdots+m_{j} T_{j},
$$

where we consider each of the terms on the right-hand side as a function of the same real variable, $H$, via (76).

To compute the Liouville measure on $Y$, recall that the symplectic form on the open subset of $X$ consisting of the Cartesian product of $X_{j_{1}}^{v}, \ldots, X_{j_{k}}^{v}$ is

$$
\Omega=d I_{1} \wedge d \theta_{1}+\cdots+d I_{k} \wedge d \theta_{k} .
$$

Lemma 5.6. Let $g=H_{1}+H_{2}$. Then

$$
\Omega^{k}=\frac{C}{(2 \pi)^{k}}\left(\prod_{j=1}^{k} T_{j}\right) d G_{1} \wedge \cdots \wedge d G_{k-1} \wedge d g \wedge d \theta_{1} \wedge \cdots \wedge d \theta_{k} .
$$

Proof. From (79) and $T_{j}=2 \pi \frac{d I_{j}}{d H_{j}}$ we get

$$
\Omega^{k}=\frac{1}{(2 \pi)^{k}}\left(\prod_{j=1}^{k} T_{j}\right) d H_{1} \wedge \cdots \wedge d H_{k} \wedge d \theta_{1} \cdots \wedge d \theta_{k} .
$$

Thus it suffices to check that

$$
d H_{1} \wedge \cdots \wedge d H_{k}=C d G_{1} \wedge \cdots \wedge d G_{k-1} \wedge d g .
$$

This follows by inverting the transformation from $H_{1}, \ldots H_{k}$ to $g, G_{1}, \ldots, G_{k-1}$ given by the defining equations for the latter. The calculation will be omitted.

Corollary 5.7. Let $1: Y \hookrightarrow X$ be the inclusion map. Then the Liouville measure on $Y$ is given by the $(k+1)$-form on $Y$

$$
\eta=\frac{2 C}{k !(2 \pi)^{k}}\left(\prod_{j=1}^{k} T_{j}\right) d H \wedge d \theta_{1} \wedge \cdots \wedge d \theta_{k} .
$$

Proof. It follows from the previous lemma and the remark that

$$
\imath^{*}(g)=2 t^{*}\left(H_{1}\right)=2 H .
$$

We can now finish our calculation: To compute $\int_{Y}\left(\mathscr{T}^{*} \hat{\varphi}\right) \eta$, use the previous expression for $\eta$ and the fact that

$$
\mathscr{T}^{*} \hat{\varphi}=\hat{\varphi}\left(F_{1}(h), \ldots, F_{k-1}(h)\right)
$$

(see (77)). Performing the $d \theta$ integrals produces a factor of $(2 \pi)^{k}$. We finally get

$$
\int_{Y}\left(\mathscr{T}^{*} \hat{\varphi}\right) \eta=C \int_{\mathscr{R}_{\nu}} \hat{\varphi}\left(F_{1}(h), \ldots, F_{k-1}(h)\right)\left(\prod_{j=1}^{k} T_{j}(h)\right) d h,
$$

which proves the formula. 


\section{On the Level Spacings Distribution}

As we stated in Sect. 1, the only rigorous approach we know of to the problem of computing the level spacings distribution is by using the Bohr-Sommerfeld quantization rules. As explained there, one needs that the BS eigenvalues exhaust the spectrum and that they approximate the true eigenvalues to a higher order than the mean level spacing. As a model problem for this we take an operator of the form

$$
D=\Delta_{0}+A,
$$

where $\Delta_{0}$ is the standard Laplacian on $S^{2}$ and $A$ is a zeroth order self-adjoint pseudodifferential operator on $S^{2}$ commuting with $\Delta$ and whose reduced symbol,

$$
H: \mathcal{O} \rightarrow \mathbb{R}
$$

satisfies the following strong assumption:

$H$ is a perfect Morse function, that is, it has only two critical points and they are non-degenerate.

This is of course very restrictive, and we cannot prove that the operator $A$ arising from a Zoll metric has this property. However, for operators of the form (81) whose reduced symbols satisfy this assumption one can compute the level spacings distribution, as we will see.

The spectrum of an operator of the form (81) forms clusters, just like a Zoll Laplacian:

$$
\operatorname{spec}(D) \subset \bigcup_{l=1}^{\infty}[l(l+1)-C, l(l+1)+C],
$$

where for large $l$ the interval $[l(l+1)-C, l(l+1)+C]$ contains precisely $2 l+1$ eigenvalues:

$$
\operatorname{spec}(D) \cap[l(l+1)-C, l(l+1)+C]=\left\{l(l+1)+\mu_{l, k} ; k=1, \ldots, 2 l+1\right\} .
$$

Introduce the distribution function of the Hamiltonian $H$ :

$$
F(t):=\text { Area } H^{-1}(-\infty, t],
$$

where the area is with respect to the Liouville measure on $\mathcal{O}$. Our assumption on $H$ implies that $F$ is a continuous function on the real line, $C^{\infty}$ on $(\min H, \max H)$. If $N, S \in \mathcal{O}$ are respectively the maximum and minimum of $H$, and if we let

$$
\check{0}:=\mathscr{O} \backslash\{N, S\},
$$

then the function $I:=F \circ H$,

$$
I: \check{\mathscr{O}} \rightarrow \mathbb{R},
$$

is the action function of the action-angle coordinates of the completely integrable system $(\mathcal{O}, H)$ (see [1]). The Hamiltonian $H$ is, on $\mathscr{\mathcal { O }}$, a function of the coordinate $I$ : there is a function $E$ on $(0,4 \pi)$ such that

$$
H=E \circ I \text {. }
$$

The following theorem says that the Bohr-Sommerfeld eigenvalues approximate the spectrum of (81) very well: 
Theorem 6.1. For every $l=1,2, \ldots$ and every $k=1,2, \ldots, 2 l+1$ let

$$
E_{l, k}=E\left(\frac{2 \pi k}{l+1}\right) \text {. }
$$

Then there exists $C>0$ such that for all $l$ and $k$

$$
\left|E_{l, k}-\mu_{l, k}\right| \leqq C / l^{2} \text {. }
$$

This result follows from Colin's work, see [12].

Let us see how Theorem (6.1) allows a straightforward calculation of the level spacings distribution $v_{2}^{l}$. More precisely we will compute, for every test function $\varphi$, the limit of

$$
\frac{1}{2 l+1} \sum_{k} \varphi\left((l+1)\left(\mu_{l, k+1}-\mu_{l, k}\right)\right) .
$$

(The normalization here differs a little from that of Definition (1.3), for simplicity of calculation.) An elementary argument shows that by virtue of (84) the limit of (85) is the same as the limit of

$$
\frac{1}{2 l+1} \sum_{k} \varphi\left((l+1)\left(E_{l, k+1}-E_{l, k}\right)\right) .
$$

To estimate this sum use the mean value theorem to write

$$
E_{l, k+1}-E_{l, k}=\frac{2 \pi}{l+1} \frac{d E}{d I}\left(c_{l, k}\right)
$$

for some $c_{l, k}$ between $\frac{2 \pi k}{l+1}$ and $\frac{2 \pi(k+1)}{l+1}$. Then (86) becomes

$$
\frac{1}{2 l+1} \sum_{k=1}^{k=2 l+1} \varphi\left(2 \pi \frac{d E}{d I}\left(c_{l, k}\right)\right) .
$$

But this is $(l+1) / 2 \pi(2 l+1)$ times a Riemann sum for the integral

$$
\int_{0}^{4 \pi} \varphi\left(2 \pi \frac{d E}{d I}(s)\right) d s .
$$

If we recall that $E$ is the expression of the Hamiltonian $H$ in action-angle coordinates, we see that we can state the conclusion as follows:

Theorem 6.2. If $H$ is a perfect Morse function, for every test function $\varphi$ one has:

$$
\lim _{l \rightarrow \infty} \frac{1}{2 l+1} \sum_{k} \varphi\left((l+1)\left(\mu_{l, k+1}-\mu_{l, k}\right)\right)=\frac{1}{4 \pi} \int_{0}^{4 \pi} \varphi\left(\frac{4 \pi}{T(s)}\right) d s,
$$

where $T(s)$ is the period of the trajectory of $H$ with action $s$.

\section{References}

1. Arnold, V.I.: Mathematical Methods of Classical Mechanics. Berlin, Heidelberg, New York: Springer 1978

2. Berry, M.: Quantum chaology. Proc. R. Soc. Lond. A413, 183-198 (1987) 
3. Berry, M., Tabor, M.: Level clustering in the regular spectrum. Proc. R. Soc. Lond. A356, 375-394 (1977)

4. Besse, A.: Manifolds all of whose geodesics asre closed. Ergeb. Math. Gren. Z. 93, Springer (1978)

5. Bleher, P.: The energy level spacing for two harmonic oscillators with generic ratio of frequencies. J. Stat. Phys. 63, 261-283 (1991)

6. Bohigas, O., Giannoni, M.J.: Chaotic motion and random matrix theories. Springer Lect. Notes in Physics 209 (1984)

7. Bohigas, O., Giannoni, M.J., Schmit, C.: Spectral fluctuations of classically chaotic systems. Springer Lect. Notes in Physics 209 (1984)

8. Brummelhuis, R., Uribe, A.: A trace formula for Schrödinger operators. Commun. Math. Phys. 136, 567-584 (1991)

9. Brummelhuis, R., Paul, T., Uribe, A.: Spectral Estimates at a critical level

10. Colin de Verdièere Y.: Sur le spectre des operateurs a bicharactéristiques toutes periodiques. Comment. Math. Helv. 54, 508-522 (1979)

11. Colin de Verdière, Y.: Quasi-modes sur les variétés Riemanniennes. Inv. Math. 43, 15-52 (1977)

12. Colin de Verdière, Y.: Le spectre conjoint d'operateurs pseudodifferentiels qui commutent: Le cas integrable. Math. Z. 171, 51-73 (1980)

13. Connes, A.: A survey of foliation and operator algebras. Proc. Symp. Pure Math. 38 (1982)

14. Dyson, F.: Statistical properties of the energy levels of complex systems I-III. J. Math. Phys. 3, $140-175(1962)$

15. Duistermaat, J.: Oscillatory integrals, Lagrange immersions, and unfolding of singularities. Commun. Pure and Appl. Math. XXVII, 207-281 (1974)

16. Duistermaat, J.J., Guillemin, V.: The spectrum of positive elliptic operators and periodic bicharacteristics. Inv. Math. 29, 39-79 (1975)

17. Guillemin, V.: Band asymptotics in two dimensions. Adv. Math. 42, 248-282 (1981)

18. Guillemin, V., Sternberg, S.: Symplectic tecdhniques in Physics. Oxford: Oxford University Press 1983

19. Guillemin, V., Sternberg, S.: On some problems in integral geometry and some related problems in microlocal analysis. Am. J. Math. 101, 915-955 (1979)

20. Guillemin, V., Sternberg, S.: Homogeneous quantization and multiplicities of group representations. J. Funct. Anal. 47, 344-380 (1982)

21. Guillemin, V., Sternberg, S.: Geometric Asymptotics. Math. Surveys No. 14, Providence R.I.: Am. Math. Soc., 1977

22. Guillemin, V., Uribe, A.: Circular symmetry and the trace formula. Invent. Math. 96, 385-423 (1989)

23. Guillemin, V., Uribe, A.: Reduction and the trace formula. J. Diff. Geom. 32, 315-347 (1990)

24. Major, P.: Poisson law for the number of lattice points in a random strip with finite area. Preprint (1991)

25. Mehta, M.: Random Matrices. 2nd ed. New York: Academic Press 1991

26. Paul, T., Uribe, A.: Sur la formule semi-classique des traces. C.R. Acad. Sci Paris 313 I, 217-222 (1991)

27. Sinai, Ya.G.: Poisson distribution for a geometrical problem. Adv. in Soviet Math. Publications of the A.M.S., 1991

28. Weinstein, A.: Asymptotics of eigenvalue clusters for the Laplacian plus a potential. Duke Math. J. 44, 883-892 (1977)

29. Weinstein, A.: Fourier integral operators, quantization, and the spectra of a Riemannian manifold. Colloques Internationaux C.N.R.S. 237, Géométrie symplectique et physique. (1976)

30. Wigner, E.: Random matrices in Physics. S.I.A.M. 9, 1-23 (1967)

31. Zelditch, S.: Kuznecov sum formulae and Szegö limit formulae on manifolds. Comm. P.D.E. 17 (1\&2), 221-260 (1992)

32. Zelditch, S.: Spectrum and geodesic flow. In: Advanced Studies in Pure Math. 21, 1-19 (1992) 